\title{
Modern Radiological Postoperative Diagnostics of the Hip Joint in Children and Adults
}

\section{Moderne radiologische postoperative Diagnostik des Hüftgelenks im Kindes- und Erwachsenenalter}

Authors

Affiliations
M.-A. Weber ${ }^{1}$, M. Egermann ${ }^{2}$, H. Thierjung' ${ }^{1}$, J. K. Kloth ${ }^{1}$

Diagnostic and Interventional Radiology, Heidelberg University Hospital, Heidelberg, Germany

2 Center for Orthopedics, Trauma Surgery and Spinal Cord Injury, Heidelberg University Hospital, Heidelberg, Germany

\author{
Key words \\ - hip joint \\ - surgery \\ postoperative imaging \\ - joint replacement \\ - osteonecrosis \\ - developmental dysplasia of \\ the hip
}

\section{Abstract \\ $\nabla$}

The assessment of bone healing and loosening of endoprosthesis material was long the primary indication for postoperative projection radiography and $\mathrm{CT}$ imaging of the hip joint following trauma and endoprosthesis implantation. With the increasing number of joint-preserving surgery, e.g. of surgical hip luxation and hip arthroscopy for the treatment of femoroacetabular impingement (FAI), high-resolution imaging of intra-articular pathologies before and after surgery has become increasingly important. In this review article, diagnostic imaging of the hip joint is presented following common trauma surgery and orthopedic surgery interventions. The imaging modalities of projection radiography, CT and MRI including direct MR-arthrography are discussed with regard to their diagnostic capability in the postoperative assessment of the hip joint. Among others topics, imaging is discussed following hip arthroplasty, following surgical hip luxation and arthroscopic interventions for the treatment of FAI, as well as following core decompression for avascular necrosis of the femoral head. Moreover, orthopedic interventions of the hip joint in children and adolescents are presented and the dedicated reporting of postoperative imaging is outlined. Key points:

\section{Correspondence}

\section{Prof. Marc-André Weber}

Universitätsklinik Heidelberg, Diagnostische und

Interventionelle Radiologie Im Neuenheimer Feld 110 D-69120 Heidelberg Germany

Tel.: ++ 49/6221/5637434

Fax: ++49/6221/5626640

MarcAndre.Weber@med.uniheidelberg.de
- Consolidation of osteotomies and position of implants should be assessed in postoperative imaging.

- MRI is useful for confirming correct articulation after treatment of congenital hip dislocation.

- Radiologically assessable complications after total hip replacement are inlay wear, loosening, dislocation, periarticular ossifications and infection.
- MRI can detect and classify pseudotumours in cases of metal-metal pairing after total hip replacement.

Citation Format:

- Weber MA, Egermann M, Thierjung H etal. Modern Radiological Postoperative Diagnostics of the Hip Joint in Children and Adults. Fortschr Röntgenstr 2015; 187: 525-542

\section{Zusammenfassung \\ $\nabla$}

Die Beurteilung von Knochenheilung und Implantatlockerung war über lange Zeit die Hauptindikation der postoperativen Röntgen- und CT-Bildgebung des Hüftgelenks nach Trauma und endoprothetischem Gelenkersatz. Mit zunehmender Zahl gelenkerhaltender Eingriffe, z. B. der chirurgischen Hüftluxation und der Hüftgelenkarthroskopie in der Therapie des femoroacetabulären Impingements (FAI) gewinnt die hochauflösende Diagnostik intraartikulärer Pathologien präoperativ wie auch in der postoperativen Bildgebung an Bedeutung. In dieser Übersicht wird die bildgebende Diagnostik des Hüftgelenks nach häufigen orthopädisch-unfallchirurgischen Eingriffen vorgestellt. Es werden die Modalitäten Projektionsradiografie, Computertomografie und Magnetresonanztomografie einschließlich der direkten MRT-Arthrografie hinsichtlich ihrer Wertigkeit in der postoperativen Diagnostik des Hüftgelenks diskutiert. Unter anderem werden die Befundinterpretation nach Hüftendoprothetik, nach chirurgischer Hüftluxation und arthroskopischer Operation bei FAI sowie nach Anbohrung einer Hüftkopfnekrose besprochen. Zudem werden kinderorthopädische Eingriffe am Hüftgelenk vorgestellt und deren dezidierte postoperative Bildbefundung skizziert. 


\section{Introduction}

$\nabla$

This review article first addresses postoperative hip imaging in children before proceeding to the topics of imaging performed following joint-preserving interventions as well as hip-replacement surgeries (hip endoprosthesis).

The underlying diseases relevant to imaging are explained, the most common surgical procedures introduced and the criteria for evaluating or assessing the results in postoperative imaging, conventional radiographs, MRI and CT are discussed.

\section{Postoperative hip imaging in children}

$\nabla$

\subsection{Slipped capital femoral epiphysis}

With an incidence rate of 2-10/100000 slipped capital femoral epiphysis (SCFE) is the most common hip disease in children and adolescents [1]. This displacement of the epiphysis against the metaphysis (typically in the mediodorsal direction) primarily affects children around the prepubescent growth spurt (from nine years old to the end of growth) and has a male-to-female ratio of $2: 1$. It should be noted that approximately $50 \%$ of cases are bilateral $[2,3]$. In most cases, the affected child is significantly overweight ( $>97^{\text {th }}$ percentile for age). The acute form is less common than the more frequently observed lenta form, thus making imaging diagnostics important particularly when patients exhibit clinically limited inner rotation (positive Drehmann sign) [2]. Radiographs show loosened epiphyseal junctions. The orthograde imaging of the femoral neck using the Lauenstein method (hip flexion around $70^{\circ}$ and abduction around $50^{\circ}$ ) is critical for diagnosing SCFE. The Lauenstein view shows a flattened lateral convexity of the epiphyseal junction with the femoral head ( $\bullet$ Fig. 1$)[1,2]$. For tilting angles up to $30^{\circ}$, therapy consists of stabilizing with wires or screws. Tilting angles above $30^{\circ}$ require corrective osteotomy, e. g. Imhäuser method, i.e. inflective and valgizing osteotomy of the proximal femur [1 - 3]. Following surgical treatment of SCFE, the radiologist's task is to sufficiently evaluate the position of implants and identify any malposition ( $\odot$ Fig. 2). It can be concluded that radiological diagnostics target the following points following the fixation of SCFE:

Report checklist

- Is there "secure" three-dimensional fixation of the epiphysis?

- Can intra-articular position of the implants be excluded?

- Treatment of the contralateral side or frequent examination thereof due to the $50 \%$ risk of bilateral epiphysiolysis of the femoral head.

- Does radiological imaging show osseous consolidation following corrective osteotomy sufficient for full weight to be placed on the joint?

- Evaluating the joint space and joint congruency.

- Follow-up examinations to exclude the development of hip necrosis following closed/open reposition.

\subsection{Perthes disease}

Perthes disease is the idiopathic pediatric osteonecrosis of the femoral head which appears between the age of five and seven and occurs at a male-to-female ratio of $4: 1$. It is bilateral in $15 \%$ of cases [4]. The key clinical symptoms are frequently limping with limited mobility of the hips and, in rare cases, knee pain. It should be noted that inguinal hip pain is the exception [5]. The extent of the femoral head involvement is classified into four Catterall grades on axial hip radiographs, group III and IV having an unfavorable prognosis and therefore requiring treatment. Risk signs highly suggestive of an unfavorable course include, among other findings, lateralization of the epiphysis, lateral calcification of the epiphysis and involvement of the metaphysis [4, 5]. Loss of mobility, flection and abduction position, abduction contracture and being overweight are clinical "head at risk signs", the collective presence thereof indicating imminent irreversible damage to the femoral head. Radiological signs of Perthes disease are visible expansion of the joint space (initial stage according to Waldenström), densification of the femoral epiphysis (condensation stage), fragmentation of the osseous core of the femoral head (fragmentation stage) and mushrooming deformation of the femoral head in the late stage [5]. When risk signs appear (particularly lateralization and lateral calcification), centering, i. e. the containment of the femoral head to prevent further deformation, by means of intertrochanteric varisation osteotomy or pelvic osteotomy according to Salter is recommended. The complication of Perthes disease is the development of prearthritic deformation such as coxa plana and coxa magna, which can necessitate joint replacement during early adulthood [4]. The goal of treatment is to preserve mobility, thereby allowing physiological regeneration to form a spherical femoral head. Should early conservative measures, particularly physical therapy, fail to bring about the desired success, the centering of the femoral head and the rotating in of the non-endangered portions of the main load zone can be attempted through surgical therapy. When it comes to prognosis, age is of major importance, with a younger age at the onset of the disease meaning better femoral "regeneration" during the repair phase [5].

The role of imaging is to provide stage classification, detect the progress and the presence of bilateral involvement as well as assess the correct position of the osteosynthesis material or the occurrence of implant failure following surgical treatment. Surgical methods for centering the hip joint in cases of Perthes disease are intertrochanteric varisation osteotomy for stage II through IV with "head at risk signs" ( $\bullet$ Fig. 3) and, if the patient is between the age of five and eight years, pelvic osteotomy, e.g. according to Salter in patients exhibiting the aforementioned indications plus limited mobility, and a valgization osteotomy ( $\bullet$ Fig. 4 ) for older patients experiencing pain, hinge abduction and fixed malalignment. In addition to femoral corrective osteotomy, pelvic osteotomy (e.g. according to Salter) has established itself as another standard method for achieving better containment of the femoral head (see also Hip dysplasia surgery with corresponding diagrams and image examples in subsection 1.4). To conclude, it is the radiologist's task to check for the following when evaluating therapy performed to treat Perthes disease:

Report checklist

- Development of the "head at risk": signs

- Checking the contralateral side in bilateral cases

(15\% incidence rate) 

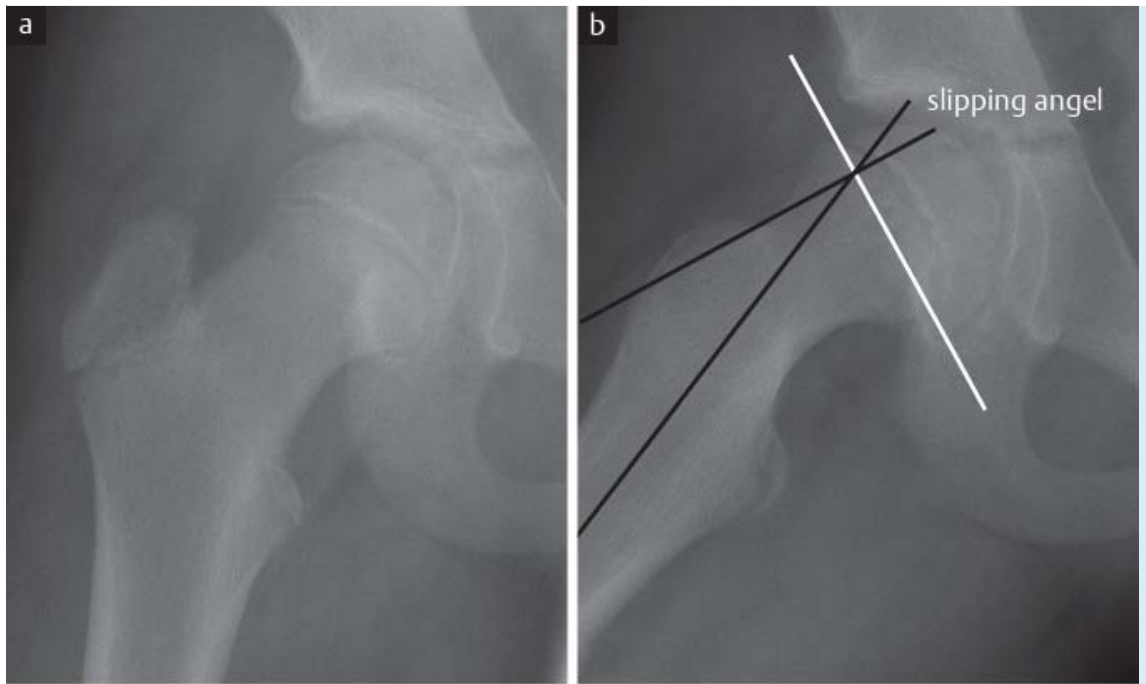

Fig. 1 10-year-old girl with slipped capital femoral epiphysis on the right side $\mathbf{a}$. The tilting angle is illustrated $\mathbf{b}$. The white line shows the basis of the epiphysis, the black lines the axis of the epiphysis perpendicular to the basis of the epiphysis and the axis of the femoral shaft. The ET- or slipping angle is $7^{\circ}$ in this example. Subsequently $\mathrm{c}$ the patient was fixated using a cannulated screw (extra-articular fixation using a single screw). Alternatively, fixation using K-wires can be performed. The follow-up images after 10 months $\mathbf{d}$ show slipping of the capital femoral epiphysis of the contralateral side. This is why the prophylactic treatment of the contralateral side is recommended. The follow-up one year later shows the bilateral fixation using cannulated screws e, f.
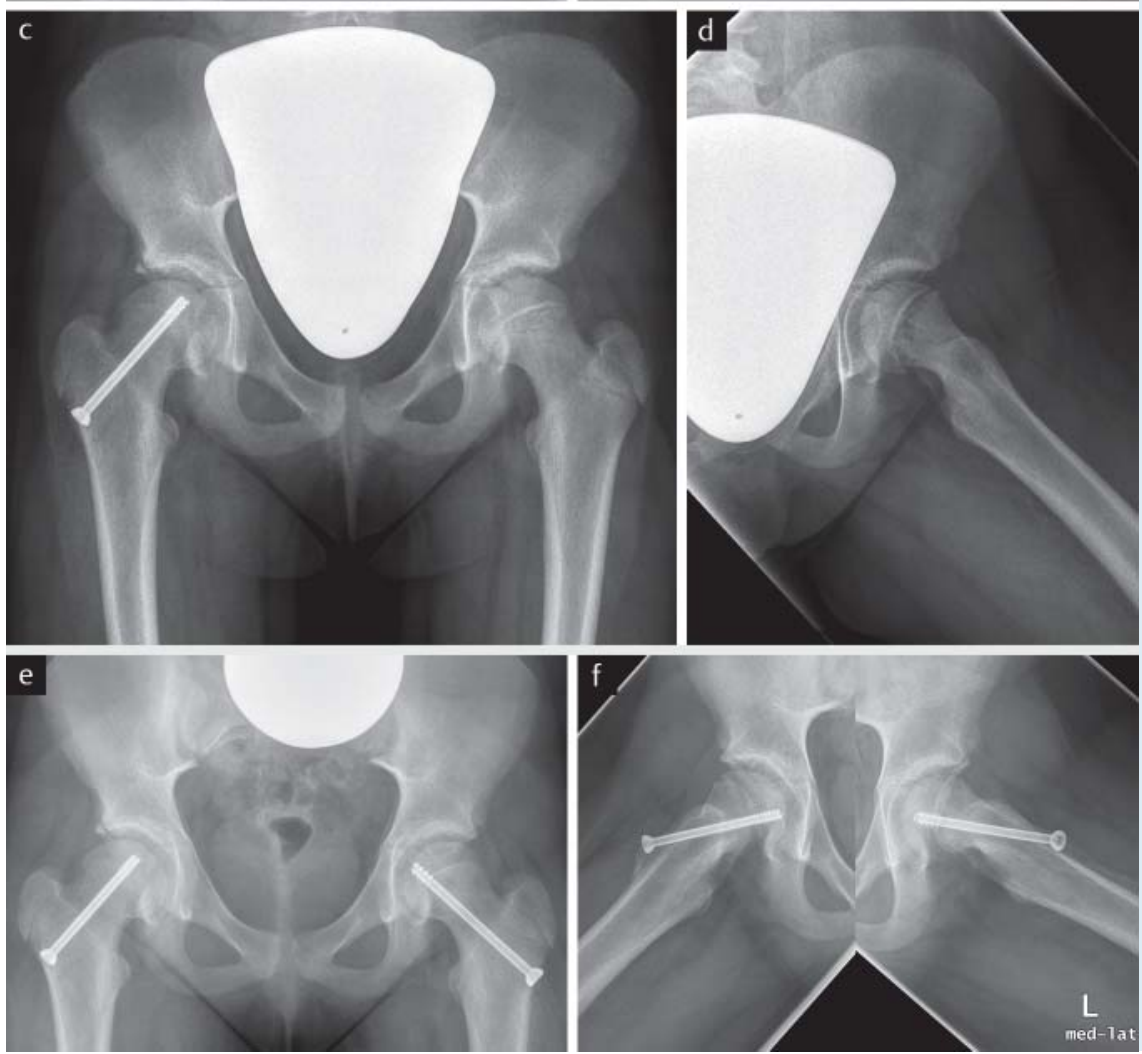

- In cases of surgical therapy:

- Have the osteotomy spaces consolidated?

- Correct position of implants. Can intra-articular position of implant material be excluded?

- Documentation of surgery success or the targeted improvement of containment

- Is implant failure present?

\subsection{Congenital hip luxation/hip dysplasia}

Dysplasia and luxation of the hip are morphological entities of various degrees [6]. Congenital hip luxation (Graf stage 3 and 4 ) is treated in-house through repositioning and setting a plaster cast (pelvic-leg cast/hip spica cast) under anesthesia with subsequent MRI for verifying normal articulation $[3,6]$. It is important that the child is sent for imaging immediately afterward to keep the anesthesia period as brief as possible and to minimize problems with movement artefacts despite the presence of a cast in newborn patients. The sequence protocol at our hospital consists of an axial and coronal localizer and, as the most important sequences, an axial and coronal T2-weighted turbo spin-echo sequence [7] as well as an axial fat-suppressed T2-weighted turbo spin echo sequence (acquisition time for this basic protocol: $5 \mathrm{~min} 20 \mathrm{~s}$ ). With the infant under continued sedation, supplemental sequences include a sagittal proton-denseweighted turbo spin echo sequence performed separately on the right and left hip ( $\bullet$ Table 1 ). The MRI protocol can optionally be concluded with a coronal proton-dense weighted turbo spin echo sequence in axial and coronal orientation (total acquisition time: $10 \mathrm{~min} 54 \mathrm{~s}$ ). The examination is performed with the small flex coil lying on the abdomen and with powered up spine matrix coil ( $\bullet$ Fig.5). 

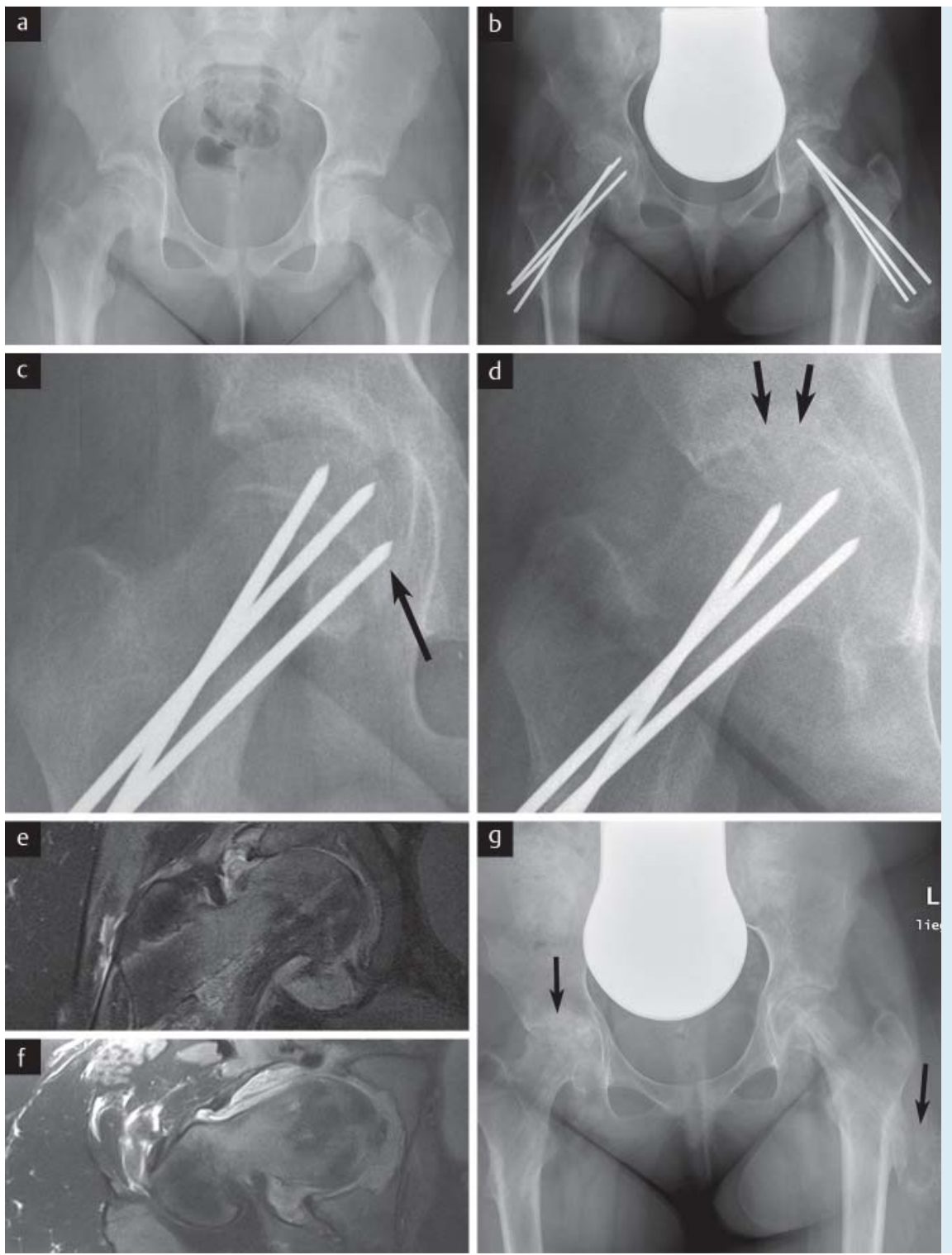

Fig. 2 9-year-old female patient with slipped capital femoral epiphysis on the right side and presentation in house seven months after treatment ex domo. Initial imaging ex domo shows epiphysiolysis on the right side $\mathbf{a}$, which was stabilized using three K-wires that were introduced through retrograde insertion b. Compared to screws, K-wires are at greater risk of positional change (migration). The enlarged picture detail shows that the medial and caudal K-wire is projecting intraarticularly with its tip (c, black arrow). At time of presentation in house there is already increasing joint space narrowing (d, black arrows) and persisting, motion-induced pain. Following implant removal, the subsequent MRI examination shows depleted cartilage layer at the acetabulum roof, joint effusion and accompanying bone marrow oedema corresponding to a severe osteoarthritis of the hip at infancy (e, coronal and $\mathbf{f}$, transverse PD-w, fs). In addition, there is ossification of the musculature on the left side at the former entry point of the K-wires (arrow, status post implant removal one year after initial medical treatment) and also severe osteoarthritis of the right hip (g, arrow).
Table 1 In-house MRI protocol for congenital hip luxation in infants (3 Tesla).

\begin{tabular}{|llll|}
\hline sequence & voxel size in mm & $\begin{array}{l}\text { measurement } \\
\text { time in s }\end{array}$ & remarks \\
\hline coronal T2 TSE & $0.6 \times 0.6 \times 2$ & 90 & BLADE \\
\hline axial T2 TSE & $0.6 \times 0.6 \times 2$ & 80 & BLADE \\
\hline axial T2 TSE fs & $0.6 \times 0.6 \times 2$ & 75 & BLADE \\
\hline sagittal PD TSE & $0.5 \times 0.4 \times 2$ & 65 & each side \\
\hline coronal PD TSE & $0.5 \times 0.5 \times 2$ & 80 & \\
\hline axial PD TSE & $0.6 \times 0.6 \times 2$ & 85 & \\
\hline
\end{tabular}

TSE: turbo spin-echo; PD: proton density; fs: fat-suppressed: sequence technique for reducing movement artifacts (also PROPELLER technique).

Conclusion: The role of radiology in treating congenital hip dislocation is verifying normal articulation through MRI.
1.4 Radiological evaluation of acetabuloplasty and triple osteotomies

Central Europe's most common congenital skeletal anomaly [6], hip dysplasia is diagnosed according to Graf score through hip sonography as part of a U3 examination [2]. For conservative therapy, Pavlik harness or similar are used, which frequently facilitate the curing of hip dysplasia $[3,6]$. Surgical therapy is necessary when therapy for treating residual dysplasia is initiated late and serves the purpose of normalizing biomechanical conditions and curing pre-arthritic deformity. Surgery is also necessary in cases of subluxations and dysplasia of neurogenic etiology. Acetabuloplasty, e. g. Dega († Fig. 6) or Pemberton acetabuloplasty, can be used for surgically treating hip dysplasia (both congenital and neurogenic) provided that the growth plate of the acetabulum (Y-shaped growth plate) is open, i.e. in children up 10 , in some cases 12 , years of age $[3,6]$. In the first technique specified, surgical procedure consists of making a gaping osteotomy above the acetabulum and inserting a bone graft from the iliac crest or other autologous bone graft (see diagram in $\bullet$ Fig. 6). While Pemberton's os- 

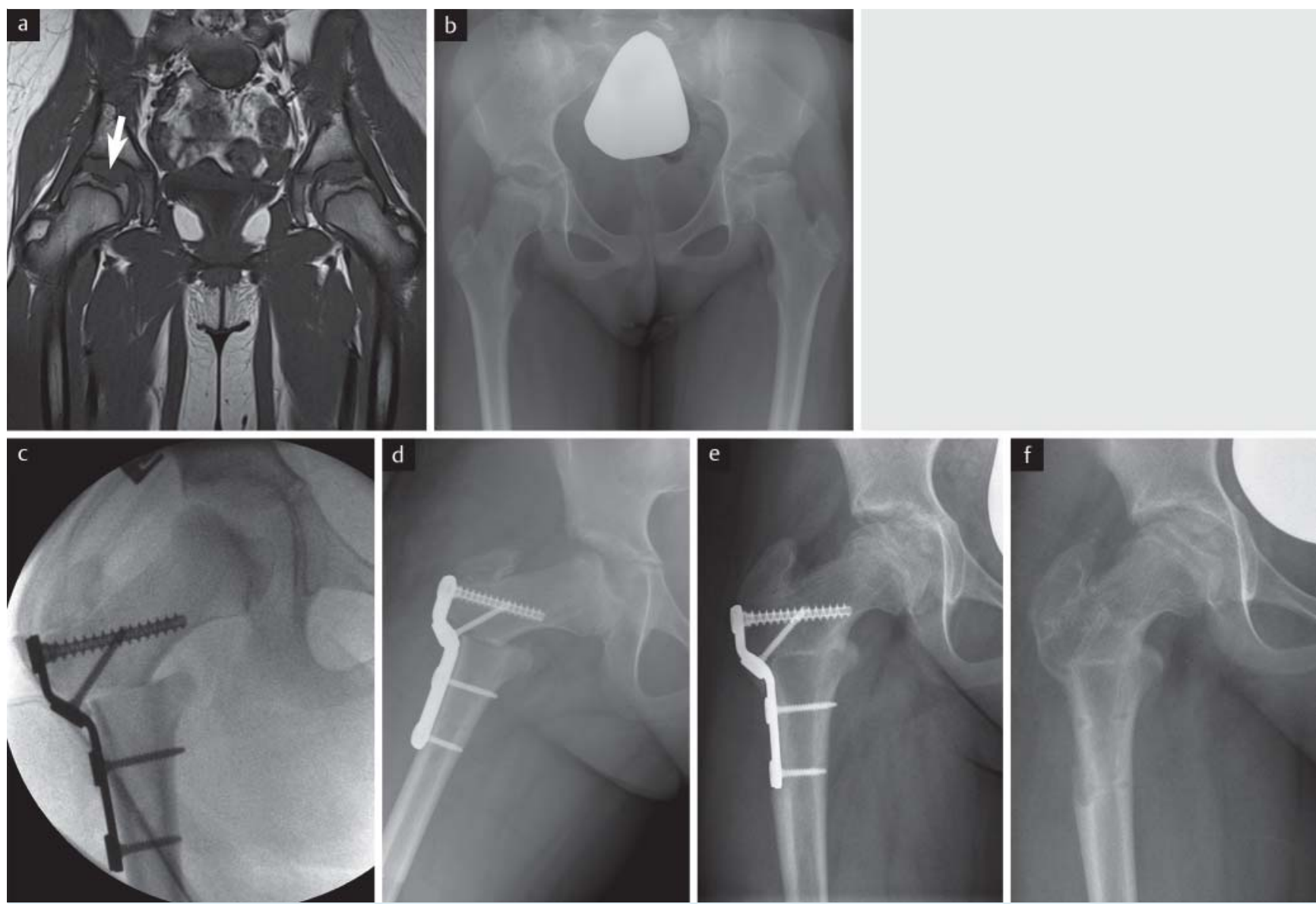

Fig. 3 7-year-old male patient with Perthes' disease. This case shows the osteonecrosis of the epiphysis more pronounced on the right than on the left side using MRI (a, coronal T1w, white arrow) and using X-rays b. Surgical treatment with varisating osteotomy $\mathrm{c}$ was performed after the patient initially presented. The goal was to improve the contact between the femoral

head and the acetabular socket (containment). The following X-ray images document the follow-up over two years with increasing osseous consolidation of the osteotomy and reconstruction of the capital femoral epiphysis d-f. teotomy differs primarily in that an aspherical osteotomy is performed, both techniques follow the same principles. In older patients with hip dysplasia, a periacetabular osteotomy (e.g. triple osteotomy) can be performed. This serves to reorient the acetabulum and normalize the deficient roofing, yet can be performed only on matured skeletons. With hip dysplasia, the labrum is typically hypertrophic and, in some cases, exhibits cystic changes with paralabral ganglion cysts. These findings are typically located in the anterior-superior quadrant [8]. The damage pattern is similar to that of femoroacetabular CAM impingement or other pathologies around the femoral head such as Perthes disease and SCFE. Triple osteotomy involves performing an osteotomy of the ischium, pubic bone and iliac bone and then rotating and stabilizing the mobilized acetabulum with $\mathrm{K}$ wires to create optimal roofing in the hip joint. Indications for this surgery are hip dysplasia and persistent ossification defect in children and adults [3]. The goal of triple osteotomy is to pivot the socket above the femoral head and to achieve optimized roofing so that the forces in the joint are transmitted over a larger surface, thus decreasing the prearthritic deformity ( $\bullet$ Fig. $\mathbf{6 g}$ ).

Following surgical treatment of hip dysplasia, the radiologist's role is to evaluate the consolidation of the osteotomy spaces $(\bullet$ Fig. 6 h, i) and the position of the material ( $\bullet$ Fig. 6 j, $\mathbf{k}$ ) (e.g.:
Is the Y-shaped growth plate injured with epiphysiodesis? Is foreign material present in the joint space?). In summary, the primary complications following surgical treatment of hip dysplasia are osteonecrosis of the femoral head, implant failure, development of pseudoarthrosis, i.e. absence of bone healing for more than six months, as well as the recurrence of malposition. Concerning pseudoarthrosis following triple osteotomy, it must be noted that this condition is frequently asymptomatic at the pubis and ischium.

\section{Imaging following joint-preserving interventions and joint-replacement surgery}

\subsection{Radiological evaluation after drilling into osteonecrosis of the femoral head}

When evaluating the course of osteonecrosis of the femoral head, is important that the correct stage is identified both before and after therapy. The MRI classification according to ARCO (Association Research Circulation Osseous) is as follows: In stage 0 all imaging techniques yield normal findings; in stage I signal changes are present in the necrotic segment; in stage II signs of T1-weighted hypointense demarcation margin and the double-line sign in T2-weighting with demarcation of the circumscribed osteonecrosis area with intact femoral head contour; in stage III subchondral 

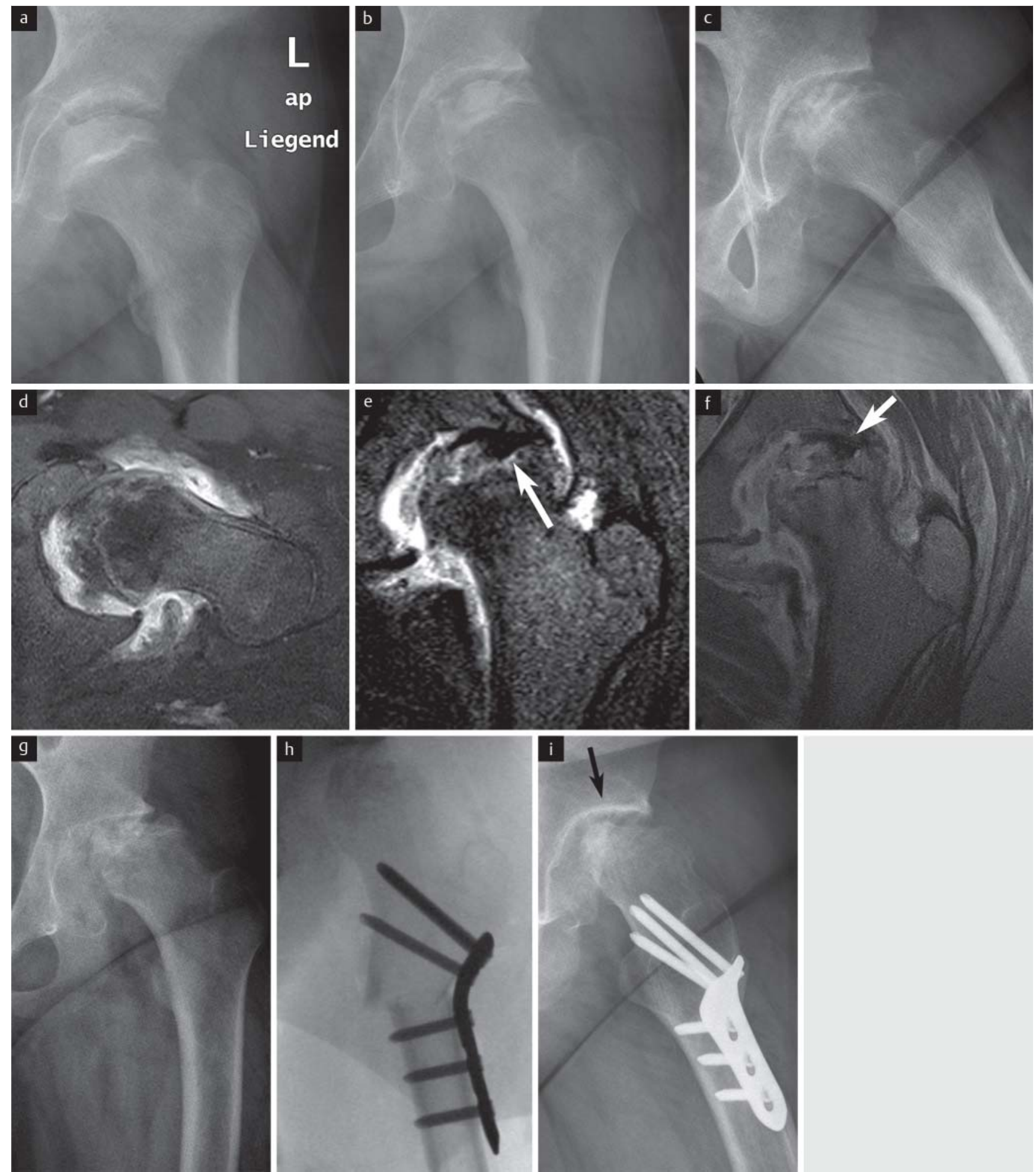

Fig. 4 10-year-old male patient with Perthes' disease, who exhibited only slight radiological progression over the first 7 months (compare a-c). After enhanced coronal T1-w fs). The projection radiograph after nine months illustrates the lateralization and the depleted joint space in terms of osteoarthritis $\mathbf{g}$. In this case the still intact areas of the femoral head were rotated to the main load-carrying areas using (arrow) a valgizing corrective osteotomy $\mathbf{h}$. The necrotic parts (see MRI) are now arranged lateral to the main load-bearing area $\mathbf{i}$. lum roof and at the opposing necrotic capital femoral epiphysis (arrows) (d, transverse, contrast-enhanced T1-w fs, e, coronal STIR, f, contrast-

fracture ("crescent sign"); and in stage IV secondary osteoarthrosis with mechanical failure of the femoral head $[5,9]$. In early stages of osteonecrosis of the femoral head, the necrotic lesion can be drilled ("core decompression" or medullary decompression, $\odot$ Fig. 7) [10, 11]. The rational for this is reducing the intraosseous pressure in the necrosis zone to thereby lower venous intraosseous hypertension. In addition, vascularization and bone growth stimulation 

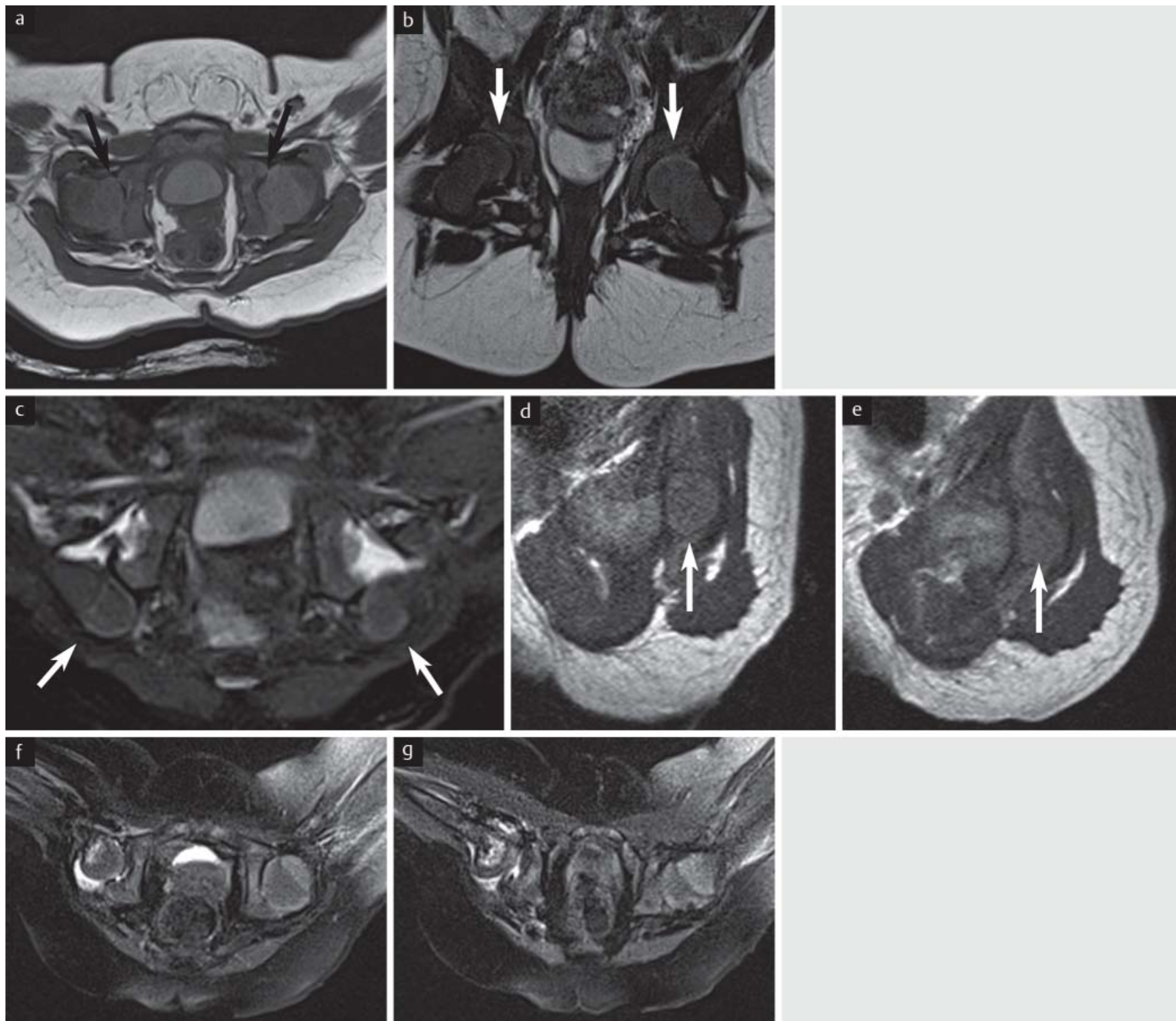

Fig. 5 Congenital hip luxation of an infant, confirmed through sonogram. MRI performed following repositioning showed normal articulation of the joints (arrows) on the T2-w turbo spin-echo sequences (a, transverse and $\mathbf{b}$, coronal). In contrast to this, are images $\mathrm{c}-\mathrm{g}$ taken from an infant following unsuccessful repositioning and bilateral persisting dorsally luxated femoral

heads (white arrows) and empty acetabulum (c, transverse T2 fs, sagittal proton-density weighting of the right $\mathbf{d}$ and left $\mathbf{e}$ hip joint). Five months later and after repeated attempts of repositioning of the right side, there was joint effusion when compared to the left side and a pathological fracture ( $\mathbf{f}, \mathbf{g}$, transverse T2-w fs in each case).

using pluripotent stem cells from blood should be performed to avert a subchondral collapse, i.e. progressing into ARCO stage III $[11,12]$. The drilling should thus be performed in ARCO stages I and II. The 15-year success rates are reported to be $90 \%$ and $66 \%$ when performed in ARCO stages I and II, respectively $[11,12]$.

The pronounced bone marrow edema without necrotic zone (transient bone marrow edema syndrome, formerly: transient osteoporosis) is no longer seen as a preliminary stage of osteonecrosis, but rather as an independent pathology of still unclear pathophysiology and self-limiting course, which can, as so-called migrating bone marrow edema syndrome, affect other bones of the joint [10, 13, 14]. Conclusion: The role of radiology following "core decompression" is to check the progress of the necrosis and monitor the success of therapy through MRI.

\subsection{Radiological diagnostics following therapy} for femoroacetabular impingement (FAI)

FAI is a frequent cause of early osteoarthrosis of the hip [15-17]. It affects $15 \%$ of the young population [8]. For evaluating the severity of the disease, it is important to bear in mind that the labral lesion, which is frequently the primary finding imaged, is only the tip of the iceberg. Pathology of the acetabulum and/or the femur usually involves cartilage damage [8]. A positive anterior labrum impingement test, i.e. a reproducible inguinal pain occurring when hip flection and inner rotation are combined, is a clinical sign of a labral lesion $[10,15,16]$. The diagnostic algorithm is comprised of medical history, clinical examination (positive inguinal tenderness, anterior labrum impingement test), radiographs and subsequent MRI by means of direct MR arthrography for evaluating the labrum and car- 

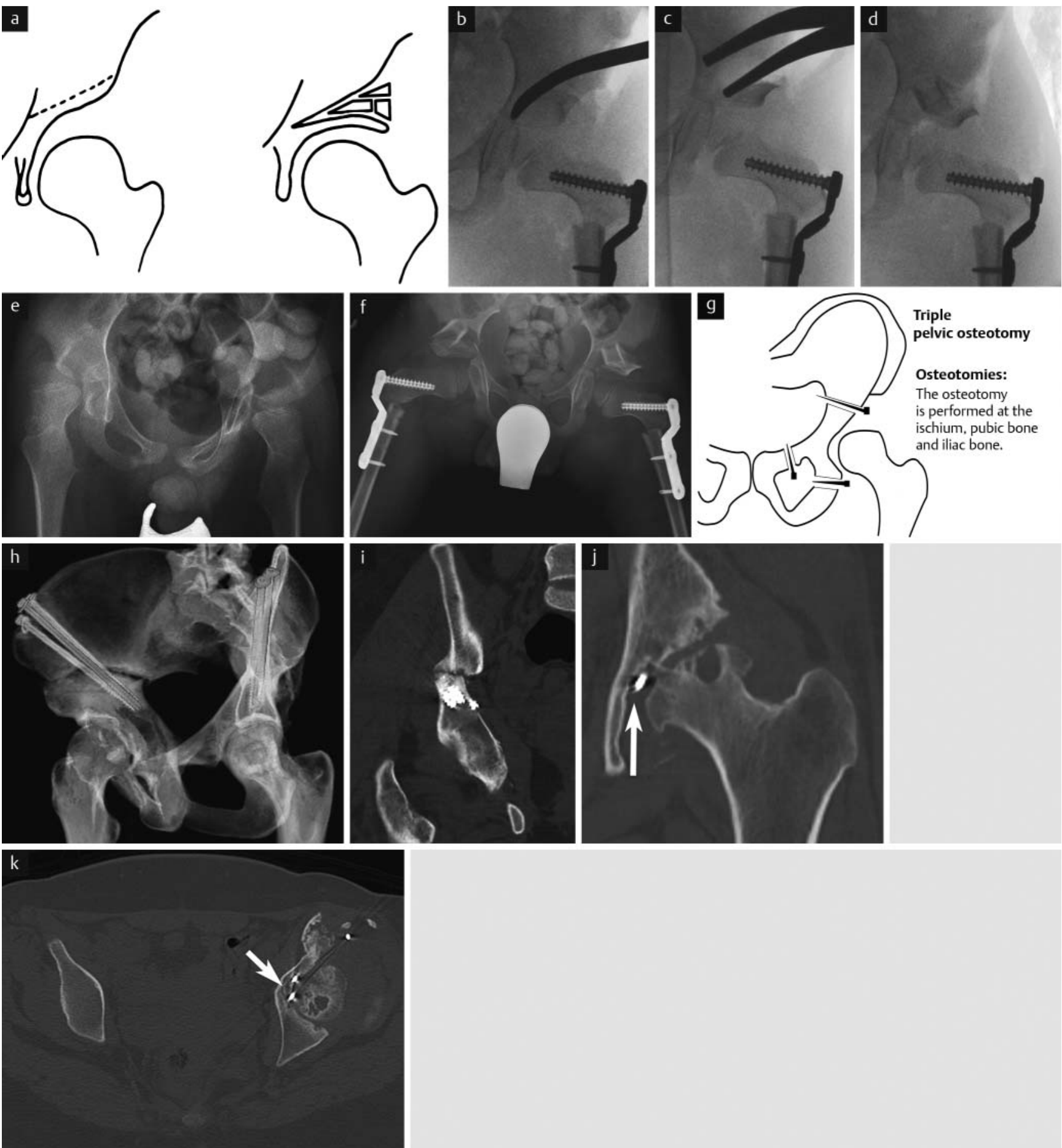

Fig. 6 7-year-old boy with infantile cerebral palsy, spastic tetraparesis and neurogenic hip luxation on both sides. The radiographic Rippstein-1 view (X-ray survey in $90^{\circ}$ flexion of knee/hip and $20^{\circ}$ abduction of the hip) shows bilaterally distinct deficient roofing with subluxation of the femoral head on the right side and bilateral fleeing and steep acetabulum roof $\mathbf{e}$. Corrective surgery with reconstruction of both hips was performed including varisation osteotomy with derotation and acetabular roof reconstruction according to Dega. Dega acetabuloplasty a is in house the standard of treatment and is performed as supraacetabular transiliac continuous osteotomy. Intraoperative $\mathrm{X}$-rays show the curved bit, which is inserted until the $\mathrm{Y}$ - shaped jointing $\mathbf{b}$, the spreading of the osteotomy using a spreader $\mathbf{c}$ and then the impact driving of the wedge of bone that has been removed from the proximal femur into the pelvic osteotomy. The wedge of bone gets pressfit jammed and the image intensifier control image shows the final situation after removal of the spreader $\mathbf{d}$. Because patients with hip dysplasia present with a valgus and malpositioning in internal rotation e, treatment is varisating and derotating $\mathbf{f}$. Schematic drawing of triple pelvic osteotomy $\mathbf{g}$. Bony non-union within the os ilium after triple pelvic osteotomy $\mathbf{h}, \mathbf{i}$ and intraarticular position of material as complication of this operation $\mathbf{j}, \mathbf{k}$. tilage $[8,17]$. FAI is caused by incomplete congruence between the femoral head and the acetabulum. In the hip, distinction is made between Pincer impingement and CAM impingement $[16,17]$, with $86 \%$ of patients having mixed forms. A definitive diagnosis should be made only on the basis of combined appropriate clinical and imaging evi- 


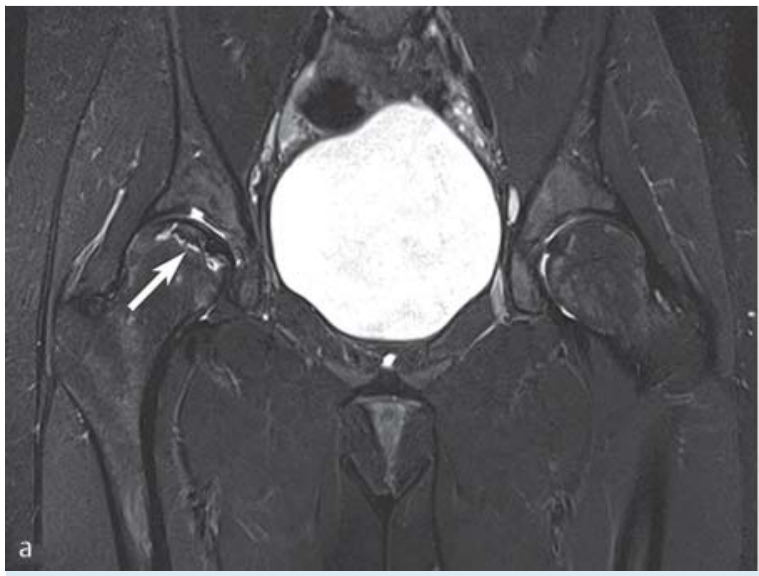

Fig. 7 17-year-old female patient status post drilling because of osteonecrosis of the femoral head. Osteonecrosis of the femoral head on the right side ARCO stage II (a, coronal STIR). Underlying condition is systemic lupus erythematosus, with the osteonecrosis of the femoral head being caused by
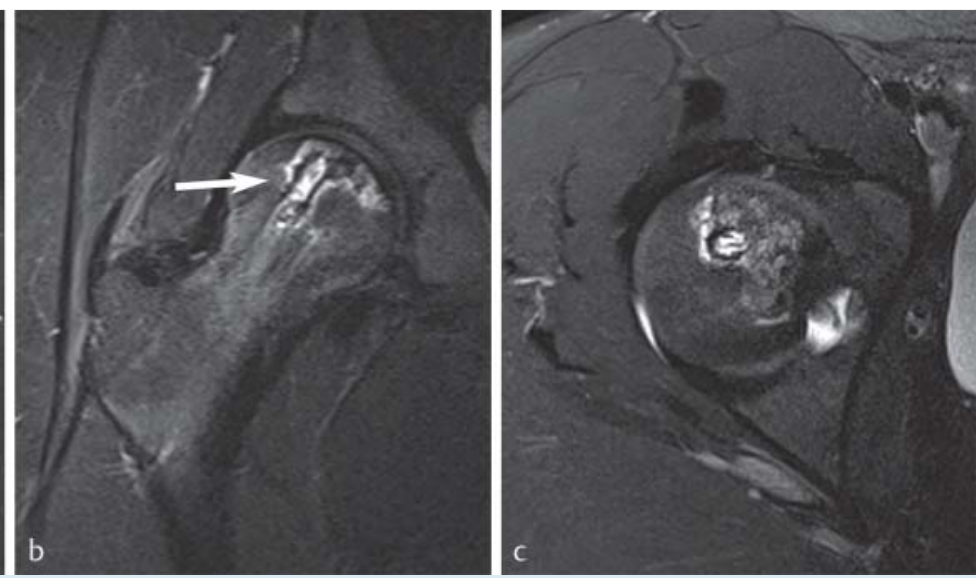

cortisone treatment. One year following drilling $\mathbf{b}, \mathbf{c}$, both cartilage and subchondral bone structure are intact (b, coronal STIR, c, transverse T2-w $\mathrm{fs})$. The arrow in $\mathbf{b}$ points at the tract of the core decompression within the right femoral neck and head. dence $[15,17]$. Only the correct roofing of the spherical head in a normal hip joint facilitates a physiological range of motion. With Pincer impingement, the excessive roofing results in premature boney contact between joint components with limited range of motion. This in turn results in damage to the labrum and joint cartilage [15]. With CAM impingement, it is the aspherical head that causes the limited range of motion and joint damage [16]. The caused is a reduced to absent offset between the femoral neck and head, a change which is referred to as "bump deformity". The term "bump" denotes the appositional boney change from which the name "cam" is derived $[15,17]$. The MR arthrography of the hip is the gold standard for detecting labral tear or detachment [18] with clearly higher sensitivity and specificity compared to normal MRI, even at 3 Tesla [18-20]. With axial slicing according the femoral neck the alpha angle is additionally determined using the Nötzli method (corresponding to femoral neck offset). This parameter measures the angle between point $A$ at which the radius exits the sphericity of the femoral head (in axial slicing) and point $B$ at which the midline through the femoral neck intersects the femoral head diameter [21].

With FAI, the goal of therapy is to surgically correct the individual pathological anatomy such that future mechanical damage to the labrum and cartilage are prevented and the impingement-free range of motion is significantly increased $[8,20]$. One method would be open hip dislocation surgery [22]. The advantage of this method is that it provides an excellent intraoperative overview of the femoral head and acetabulum, allowing FAI pathologies to be visualized and treated laterally and dorsolaterally owing to the $360^{\circ}$ joint exposure. Complications are hemorrhage, infection, overcorrection, risk of creating a bony predetermined breaking point and joint capsule adhesion. Another disadvantage of open hip dislocation surgery is the risk of pseudoarthrosis of the necessary trochanter osteotomy due to the constant muscular tension on the major trochanter by the gluteus medius and minimus muscles [8, 22]. A more minimally invasive alternative is hip arthroscopy (hip ASC) for treating localized pathologies, particularly at the anterior-superior head-neck junction $[8,23]$. The advantages of this method are that it protects soft tissue and requires significantly shorter rehabilitation. Therapeutic labral resection, reduction or short-distance refixation are also possible with this method. Complications of hip ASC are likewise development of joint capsule adhesion, in particular, however, a transient but persistent anesthesia in the thigh in the innervation region of the pudendal nerve. In addition, there is a slight risk of traction damage at the sciatic nerve, since arthroscopy requires pronounced hip extension to allow instruments access to the joint space. The disadvantages of hip arthroscopy are reduced intraoperative visibility and decreased verifiability of correct resection [8, 24]. The radiologist thus has the important task of verifying post-arthroscopic success [20]. Pathological morphologies are arthroscopically trimmed back using shavers and spherical burrs ( $\bullet$ Fig. 8). Extensive or complete labral resection provides poorer clinical results than labral refixation. The goal is thus to reconstruct, and in the case of detachment, secure the labrum, e.g. through the implantation of anchors [8]. In this case, the radiologist must check that the anchors are properly positioned post-surgery. New or persistent labral lesions can also be successfully imaged and evaluated postsurgery in MR arthrography and radial sequences [8].

Post-surgical evaluation following treatment of FAI and labral pathologies is based on the following points, and the radiologist should address the following questions in his or her report [20]:

Report checklist

- Was the offset of the head-neck junction sufficiently restored?

- Was a "bump" overlooked, i. e., is there residual asphericity, e.g., dorsal or medial?

- Is there progress in cartilage damage over time?

- Are the anchors positioned correctly following labral refixation?

- Are there residual loose bodies present following arthroscopy?

- Has new labral damage appeared?

- Can a temporary instability or predetermined breaking point in the femoral neck be assumed due to an overcorrection? 

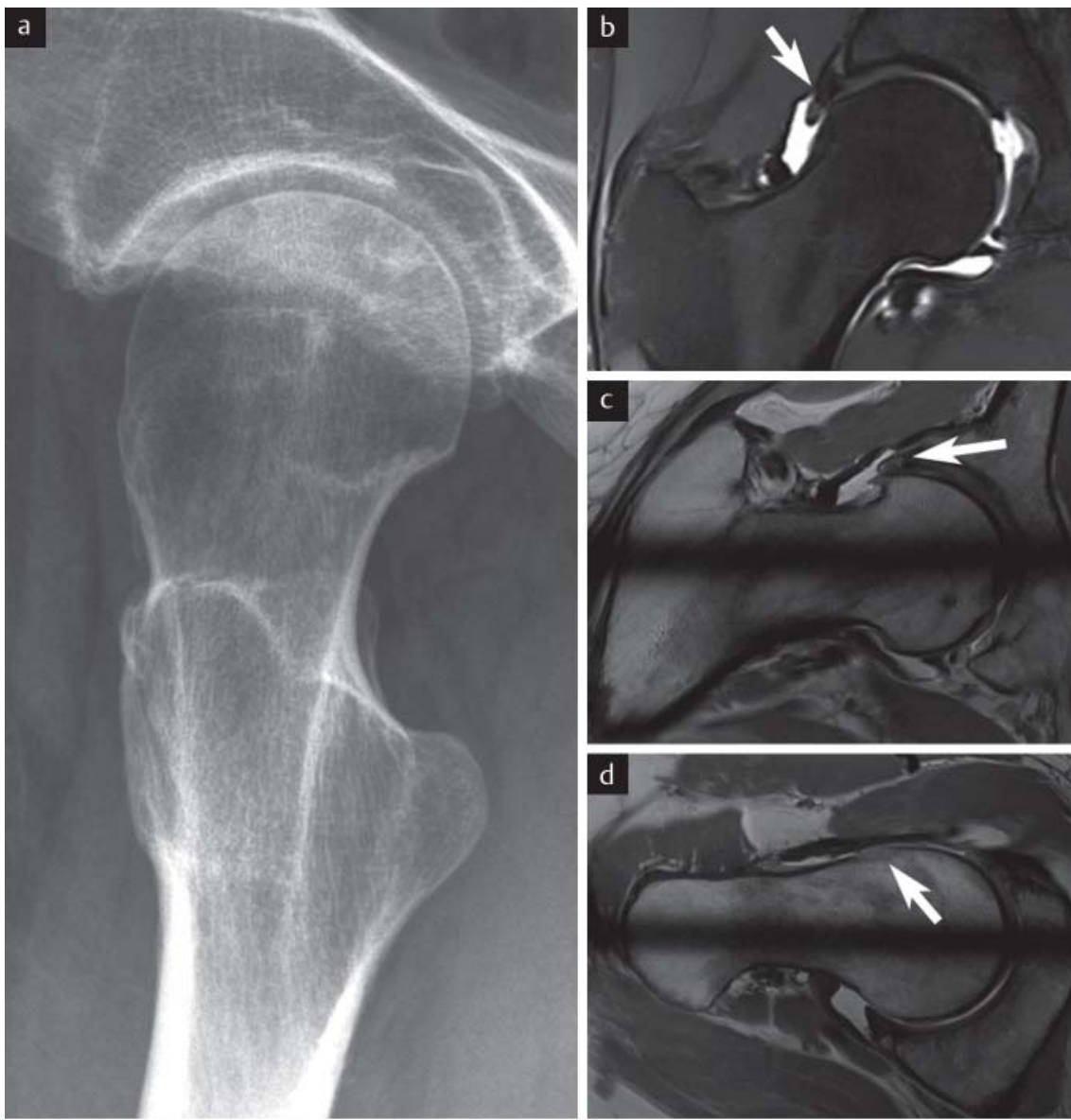

Fig. 8 Preoperative Lauenstein view of a 26-yearold female with CAM impingement a. MR-arthrography shows in addition to the bump at the headneck junction (arrow in $\mathbf{d}$ ), which is already radiographically visible, the labral lesion (arrows in $\mathbf{b}$ and c; b, coronal PD-w fs, c and d, radial PD-w sections perpendicular to the femoral neck axis). Intraoperative images of an arthroscopic bump resection in case of CAM impingement $\mathbf{e}$. In case of CAM impingement the bony asphericity at the head-neck

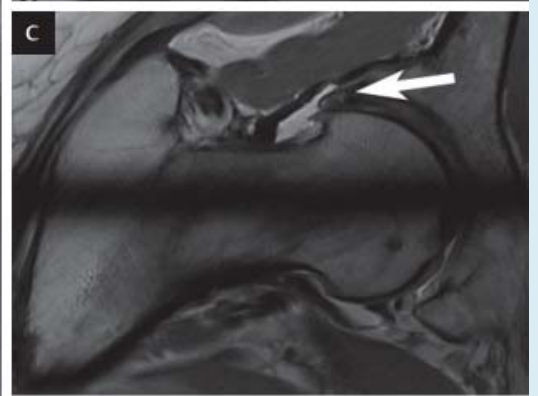
junction is removed. The Lauenstein view illustrates the removed bump and the restoration of the sphericity $\mathbf{f}$.
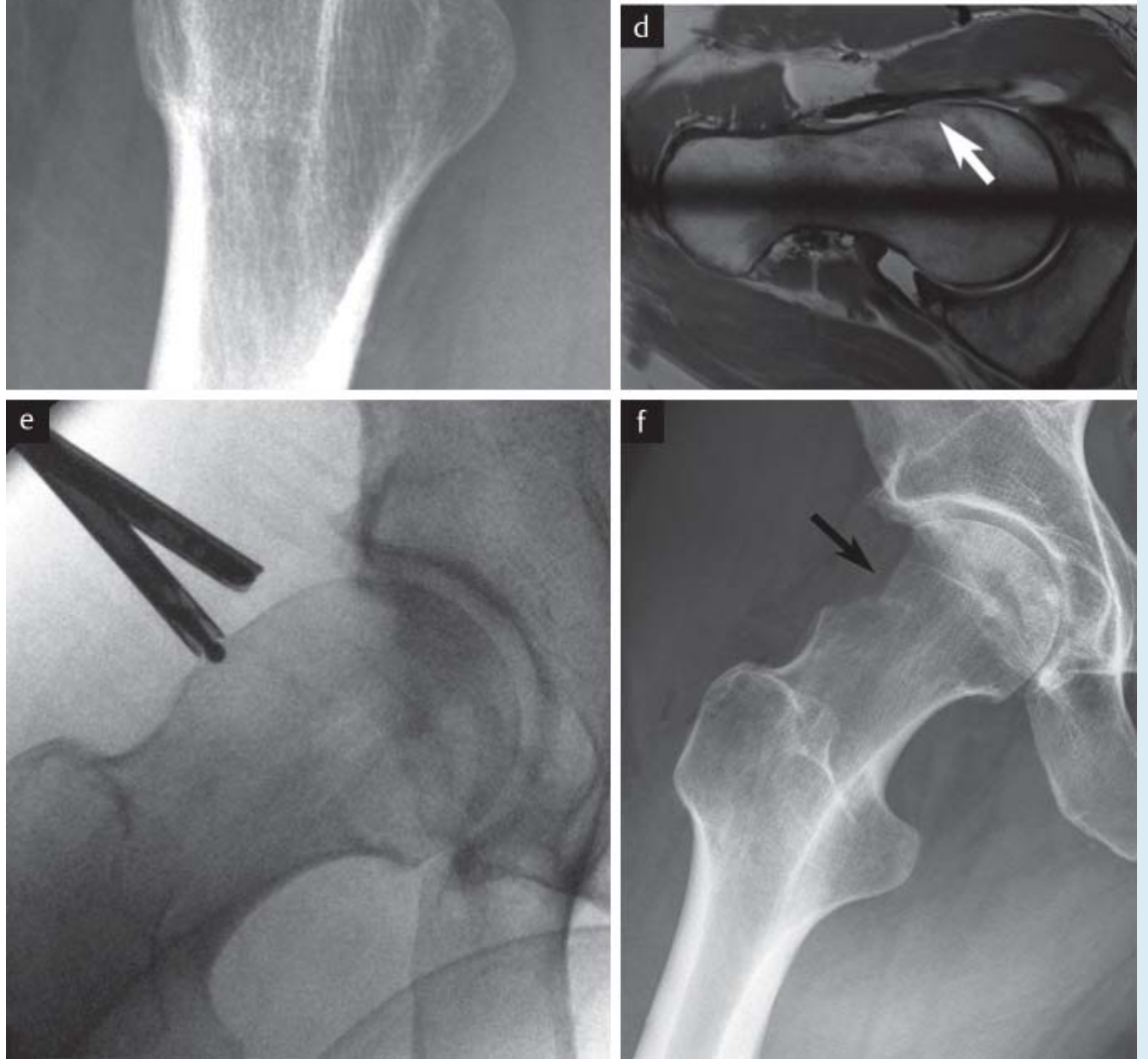

\subsection{Imaging following hip endoprosthesis}

There has been steady increase in the number of joint replacement surgeries in the hip and knee joints as well as the shoulders [25, 26]. In Germany more than 300000 joint replacement surgeries are now performed annually, with 158000 artificial hip implantations being performed in 2008 [26]. Interestingly, there are no hard indications for hip endoprosthetic surgery [27]. In addition to frequent osteoarthritis (degenerative, post-traumatic and dysplastic), osteonecrosis of the femoral head and femoral neck fracture are typical indications for a total endoprosthesis (TEP) of the hip. Not only radiological changes, but also movement restrictions and limitations in everyday life accompanied by duration and intensity of hip pain are important factors when determining whether this therapy is indicated [27]. Hip endoprostheses can be divided into hemiprostheses (replacement of the femoral head and femoral neck, e.g. duo-head prosthesis) and total endoprostheses with replacement of acetabulum, femoral head and femoral neck according to the degree of replacement $[28,29]$. Superficial replacement involving replacement of the acetabular and femoral head surfaces must also be mentioned. However, this solution is associated with poorer long-term results and is thus of subordinate clinical relevance. Furthermore, hip prostheses are commonly subdivided according to implantation technique into cemented hip TEP, especially in 
osteopenic bones and older patients, and non-cemented hip TEPs. The later solutions are implanted primarily in younger, active patients, since they can be replaced with less loss in bone mass $[28,29]$. Special forms include tumor prostheses and revision prostheses, the modularity of which allows them to adapt individually to the bone defects in the femur and pelvis [28].

When it came to long-term results, cemented prostheses were the long-reigning gold standard, showing very positive results especially in nation-wide registers for the Scandinavian countries [30]. Cement-free prosthetic systems have been steadily approaching the long-term results of their cemented counterparts and have become increasingly popular around the world because of the advantages they offer with regard to implantation and replacement [11, 29]. Today, cement-free and cemented prostheses have comparable service lives. International comparison has shown clear differences in the use of these two anchoring systems. In no way should cemented prostheses be labeled as "old" and cement-free as "modern". The choice between cemented and cement-free treatment is made on the basis of age, bone quality, concomitant diseases, previous surgery, etc. [28, 31, 32]. For cement-free systems, the pelvic bones and femur are prepared with surgical burrs and bone rasps, and the implant is inserted using the press-fit technique (i.e. adjusting the pressure of the implant going into the bone without using external materials). High primary stability is the prerequisite for secondary biological anchoring in the bone (osteointegration) [11]. Serving as sockets are widely distributed hemispherical and rough surfaced systems which are anchored in the acetabulum through press-fit with or without additional screws. Threaded cups are also still commonly used. For cement-free shafts, proven systems with meta-diaphyseal anchoring are available that can be described as long-shaft systems. The short-shaft prostheses are based on a purely metaphyseal anchoring, and the surgical technique demands preserving the medial femoral neck. In the case of standard interventions, immediate mechanical loading is permitted for both cement-free and cemented prostheses [33].

In terms of tribological pairing, hip total endoprosthesis can be divided into ceramic, metal and polyethylene sockets, which can in turn be combined with ceramic or metal heads ( $\bullet$ Fig. 9a-e). The goal is optimal tribological pairing of the head and socket to keep the constantly present wear to an absolute minimum [11]. The softer material always wears off. In the case of polyethylene-metal pairing, the biologically active polyethylene exhibits an average annual wear of $0.2 \mathrm{~mm}$. The wear products activate osteoclasts via complex cascades and result in sometimes pronounced bone defects around the embedded prosthesis [29]. Metal prostheses entail the problem of metal particles resulting from wear. These particles can lead to elevated metal ion values on the systemic level and be accompanied by the development of localized pseudotumors (see also subsection "Pseudotumors" further below) $[11,34,36]$. Ceramic-ceramic tribological pairings involve no appreciable wear, thus making these the preferred pairings for younger patients [11]. However, ceramic-ceramic pairings do entail the risk of inlay breakage [29], resulting in their having in some cases higher revision rates than the pairing consisting of a ceramic head and highly cross-linked polyethylene inlay. During immedi- ate postoperative evaluation, the radiologist's job is to detect or exclude peri- and postoperative complications such as fracture, malposition and prosthesis luxation [11, 36]. A correctly positioned prosthesis will have a socket inclination angle between $35^{\circ}$ and $45^{\circ}$ and anteversion between $10^{\circ}$ and $15^{\circ}$ [11]. The inclination angle is measured at a line between the Kohler's teardrops and the socket entrance plane $[11,37]$. Socket anteversion is defined through the angle between the socket entrance plane (i.e. of the tangent at the anterior and posterior edge of the socket on CT slices) and the sagittal body axis (e.g. of the parallel lines to an anterior-posterior oriented line running precisely between the two iliac crests) $[11,38]$. Various techniques (Lewinnek, Widmer, Law or Pradhan) can be used for indirectly estimating anteversion through the ellipsoid representation of the socket [39]. In postoperative imaging, it is necessary to distinguish fractures from osteotomies (e.g. shortening osteotomies on the femur). The radiologist should also indicate whether the components are over- or undersized. This is visible, for one, in the correct restoration of patientspecific biomechanical conditions with femoral offset and leg length. With cement-free shafts, the implant size is correct if there is sufficient cortical bone contact. The postoperative radiolucent margin is a periprosthetic bright area visible in non-contrast radiographs which results from a disparity between the surgically milled bone and prosthesis position. With the osseointegration of the cement-free prosthesis, this margin usually decreases significantly over time [28].

Over the long term, the following complications can arise with hip endoprostheses: inlay wear or failure ( $\bullet$ Fig. $\mathbf{9 f}-\mathbf{j}$ ), septic or aseptic loosening ( $\bullet$ Fig. 10), as well as infections $[25,29,40]$ and the sinking of the prosthetic shaft into the femur ("subsiding") [41]. Careful evaluation of the head position over a series of radiographs can prevent the need for socket replacement in the event of inlay failure. Radiological follow-up with conventional radiographs (low pelvic overview and axial view of hip) every two years is therefore advised. Other problems are heterotopic ossification (paraarticular ossification, PAO) or pseudotumors as well as wear granulomas. The key to diagnosing loosening is the appearance of a radiolucent margin of over two millimeters progressing over time as well as the migration of the prosthesis, e.g. the sinking of the shaft or the protrusion of the socket $[11,28,40]$. The appearance of a radiolucent margin only in the proximal femoral area of the prosthesis (in the intertrochanteric region) initially has no clinical relevance and physiologically results from mechanical loading and load effect further distal [28]. With cemented systems, the appearance of a radiolucent margin especially at the anchoring point is clinically relevant. Supplemental scintigraphy can be performed when radiographs as primary imaging modality and $\mathrm{CT}$, which is the most sensitive at detecting the appearance of radiolucent margins, are not sufficiently conclusive [28]. The diagnostic value of these modalities, however, is undisputed, particularly in the first one or two years following implantation. Implant migration or positional change as well as breakage of components or the surrounding cement are other sensitive parameters for diagnosing a loosening. [29]. Thorough evaluation should therefore always include comparison with previous images $[11]$. Periprosthetic fractures ( $\bullet$ Fig. 11a-d) may occur in- 

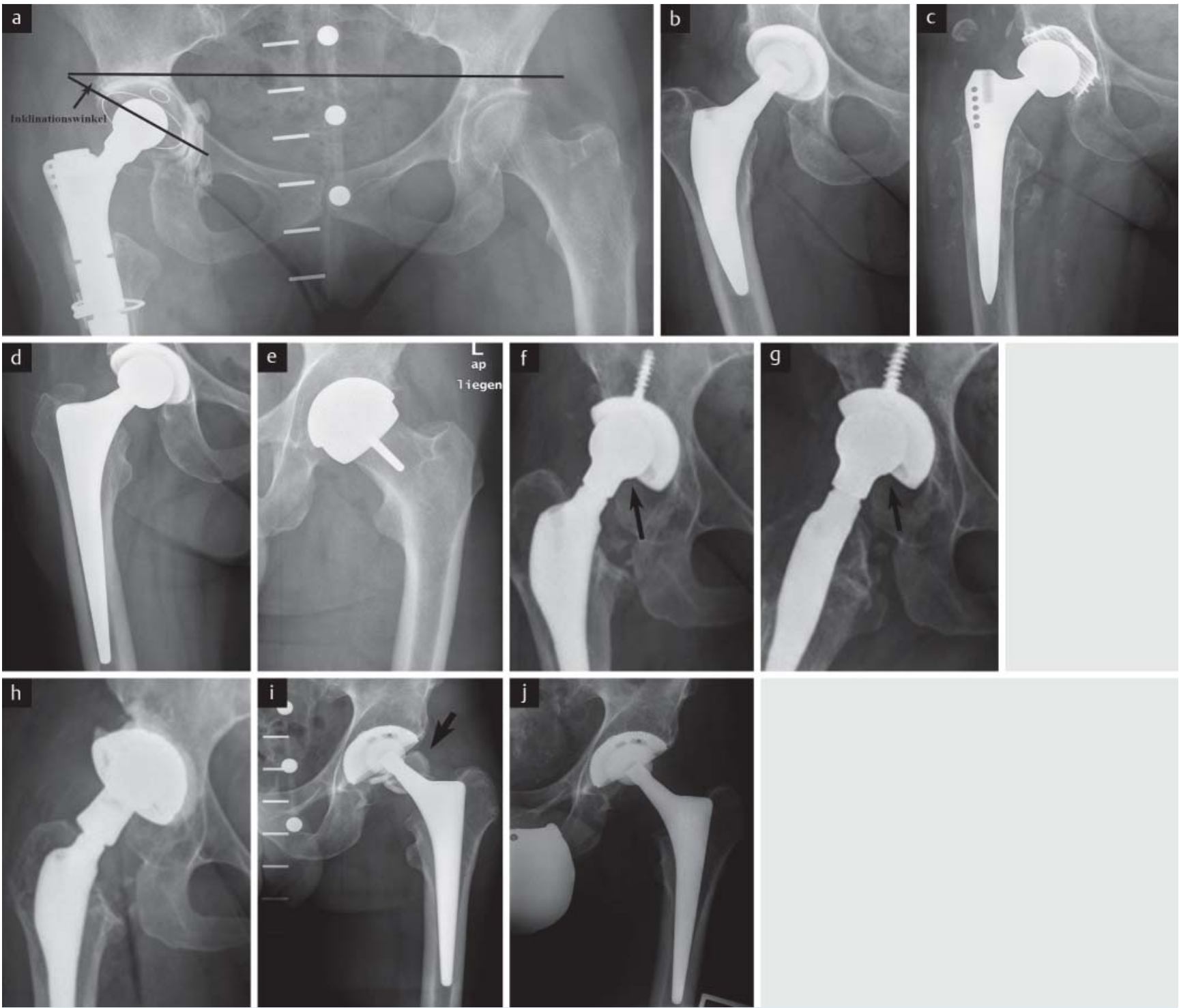

Fig. 9 Examples of a polyethylene socket with metal head. The ring marks the position of the non-radiopaque polyethylene (PE) socket and the black lines demonstrate the measurement of the inclination angle a. Examples of different combinations of artificial hip implants: ceramic head, PE inlay, press-fit cup b, metal head, PE inlay, threaded cup c, metal head, PE inlay, press-fit cup d, total surface replacement prosthesis with metal-metal pairing components e. Inlay failure in a 54-year-old woman with total hip endoprosthesis on the right side implanted 15 years before $\mathbf{f}, \mathbf{g}$. The most important imaging clue is the metal head brought out of centre, because the depleted polyethylene inlay is not radiopaque (black arrows). Because in this case, the head was reaming the socket, a complete change of the socket of the prosthesis to a cemented metal cup and also a change of the head and an extension of the neck of the prosthesis were performed $\mathbf{h}$. Failure of the ceramic inlay (arrow in i) and intact ceramic-on-ceramic bearing total hip arthroplasty were imaged prior to this $\mathbf{j}$. traoperatively, following surgery during mobilization and as a result of trauma. Avulsion fractures are frequently observed on the greater trochanter, the same being true of oblique or spiral fractures on the femoral shaft. Acetabular fractures around the implanted cup occur more rarely. When planning therapy, diagnosing or excluding a loosening of the prosthesis is crucial. A loose prosthesis must be replaced. In cases of doubt, supplemental CT should be performed liberally [40]. Periprosthetic fractures detected intraoperatively through fluoroscopic examination are treated immediately by means of cerclage.

\section{Infections}

Prosthesis-related infection is a formidable complication, appearing in $0.5-1.0 \%$ of patients worldwide. The risk factors are advanced age, obesity, diabetes mellitus and immune suppression. Distinction is made between early infections, occurring within six weeks post surgery, and late infections [28, 42]. Early infections can be treated through attempted preservation of the prosthesis by means of debridement and purging. Late infections necessitate a single or even double implant replacement. The radiologist needs to indicate whether the infection process has made contact with the prosthesis ( $\bullet$ Fig. 11e-f) or whether a periosteal reaction or osteolysis with blurry edges is present $[11,29,40]$. Inversion recovery sequences such as STIR are better suited 

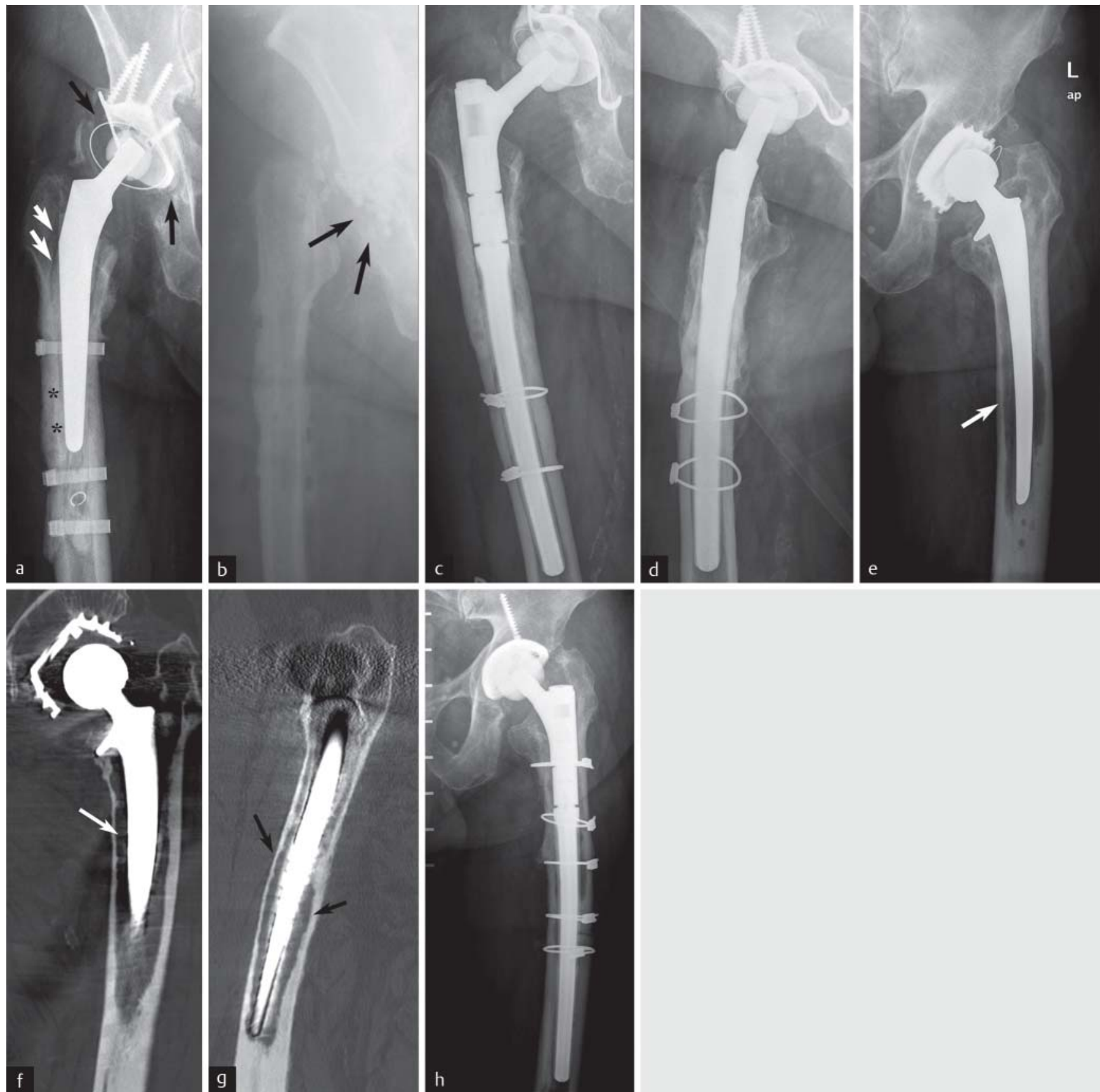

Fig. 10 Example of septic shaft loosening (a, white arrows) with osteitis $\left(^{*}\right)$ and luxation of the inlay (black arrows). A two-stage revision was carried out including removal of the prosthesis and Girdlestone arthroplasty $\mathbf{b}$. The black arrows point at applied antibiotic chains, typically gentamycin. With no evidence of ongoing infection, the change to a prosthesis with a long shaft was performed $\mathbf{c}$, $\mathbf{d}$. Examples of pronounced loosening of the prosthesis shafts with large osteolysis margins (arrows) at projection radiogra-

phy e and computed tomography $\mathbf{f}, \mathbf{g}$. Treatment was performed that involved switching to a prosthesis with a long shaft $\mathbf{h}$. This example illustrates the disadvantage of cemented prostheses, which necessitate complete removal of the cement when being replaced. In this process, the proximal femur has to be fenestrated and opened. This necessitates the implantation of cerclage cables, resulting in a higher risk of ischemic osteonecrosis and prolonged non-weight-bearing period.

than spectral fat saturation, while T1- and T2-weighted (turbo-) spin echo sequences are better suited than gradient echo sequences at decreasing the metal artifacts of the hip endoprosthesis and detecting periprosthetic bone and soft tissue changes as well as fluid collections [36, 42, 43]. The absence of joint effusion has a high negative predictive value for an infection of $96 \%$ [36]. Because of the applicability of MRI, nuclear medicine methods such as scintigraphy or positron emission tomography (PET) are used less frequently for these clinical problems [28].

\section{Pseudotumors}

Pseudotumors appearing with polyethylene inlay are called abrasion granulomas ( $\bullet$ Fig. 12a-c). The abrasion debris of the polyethylene inlay is engulfed by macrophages, which accumulate in foreign body granulomas and usually have a density of 30 Houndsfield units in CT. In MRI they appear 

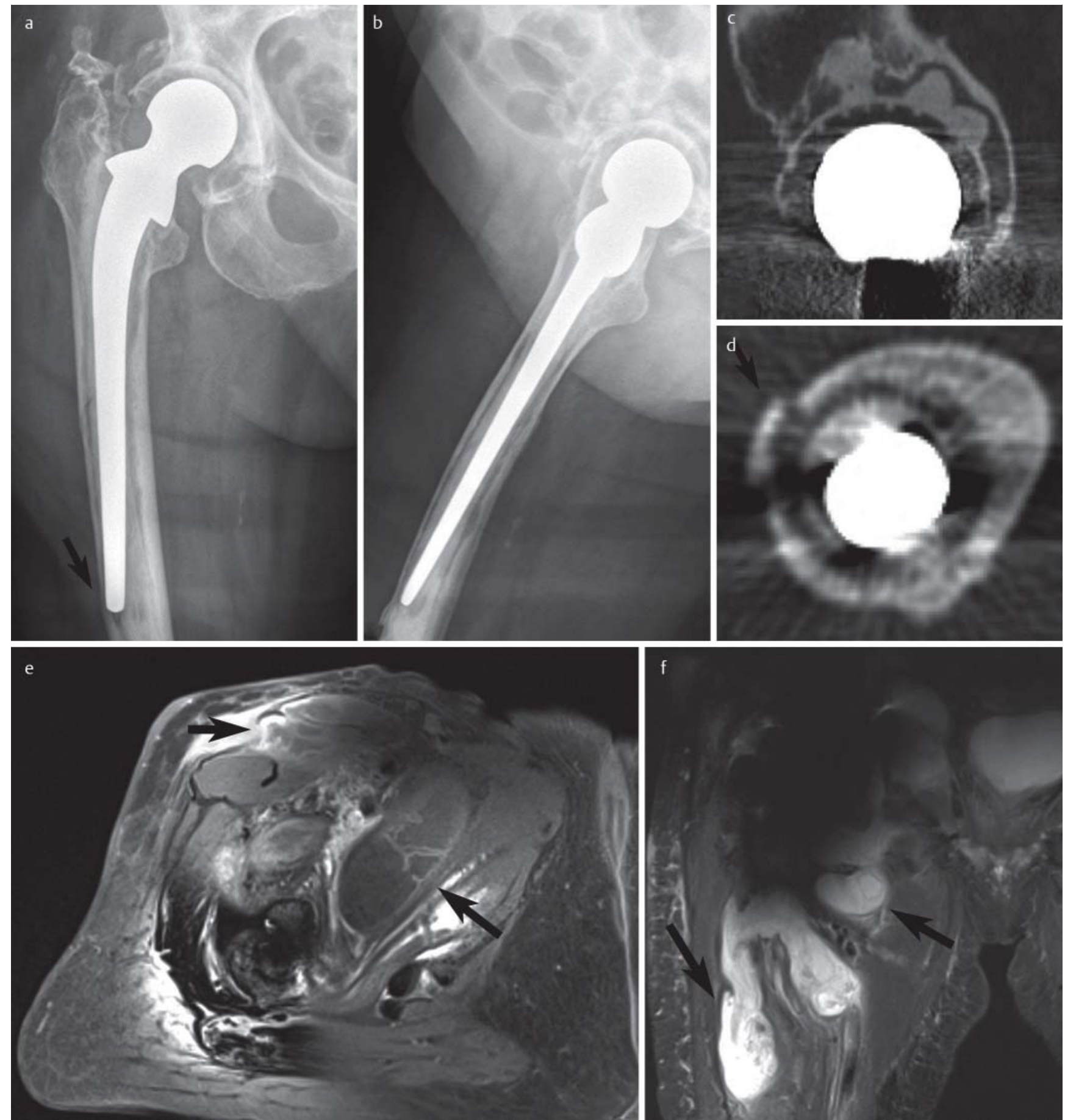

Fig. 11 Periprosthetic fracture as complication following hip endoprosthesis $\mathbf{a}, \mathbf{b}$ with break-out of the shaft at the middle third of the femur (black arrow) and depiction of the cup loosening and the periprosthetic fracture at

computed tomography c, d. Prosthetic joint infection at MRI, the fluid collection (arrows) contacts the prosthesis material (e, transverse, contrastenhanced T1-w fs, f, coronal STIR). hypointense and slightly hyperintense in relation to the muscle in T1- and T2-weighted sequences, respectively, with T2-weighting showing a hypointense line to the bone marrow, and typically involve no perifocal bone marrow edema [36, 40, 42]. With metal-metal tribological pairings, the terms cyst, ALVAL (aseptic lymphocytic vasculitis-associated lesion) or ARMD (adverse reactions to metal debris) are used synonymously with pseudotumor. These cases usually involve elevated metal ions in serum [11, 35]. The patients are usually below the age of 50 and have under- gone joint replacement surgery with metal-metal tribological paring (e.g. surface replacement) [34]. The incidence rate is $1-4 \%$ with a peak appearing four to five years following TEP implantation. Pseudotumors frequently expand into the iliopsoas muscle $[11,34]$. The key to diagnosis is detecting a space occupation in connection with the hip joint of the prosthesis and, in the case of metal-metal pairings, solid components and/or a low-signal wall in T2-weighting ( Fig. 12d-j) [42, 43]. Multiple grading systems for soft tissue changes occurring with metal-metal prostheses have 

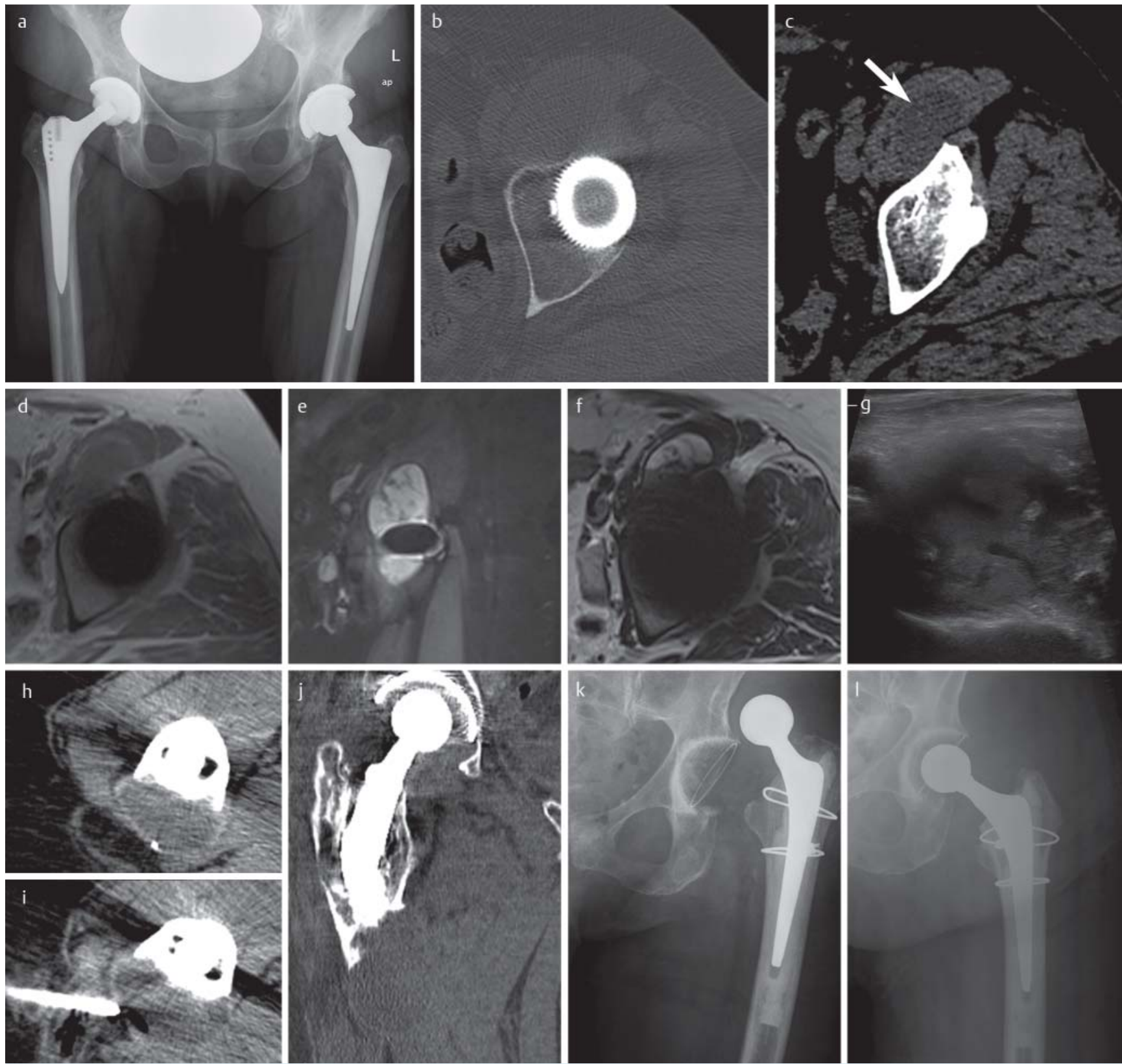

Fig. 12 Pseudotumor in a 62-year-old female patient post status metalmetal pairing of the left hip six years before $\mathbf{a}-\mathbf{c}$. Abrasion-induced synovialitis and effusion with release into the iliopsoas bursa (type 1 pseudotumor) visible on MRI (d, transverse T1-w, e, coronal STIR, f, transverse T2-w) and ultrasound $\mathbf{g}$. Revision surgery involving changing from the Metasul inlay to a polyethylene inlay and to a ceramic head was therefore indicated.
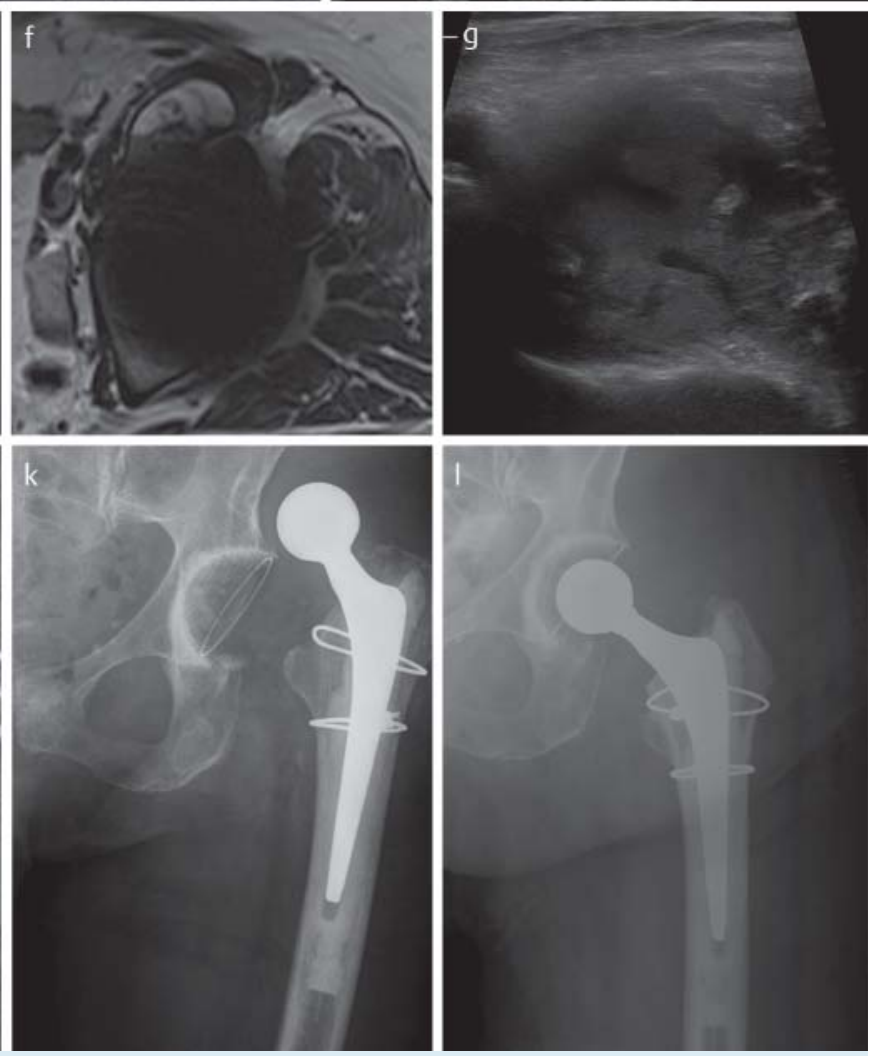

Pseudotumor in a 71-year-old female patient with implanted total hip arthroplasty and space-occupying lesion dorsal and medial to the femur as well as erosion and lysis of the cortex of the femoral shaft. A granuloma due to abrasion of the polyethylene inlay was histologically verified using CTguided puncture $\mathbf{h}-\mathbf{j}$. Luxation of total hip arthroplasty cranially in a 84year-old female patient and status post proper repositioning $\mathbf{k}, \mathbf{l}$. been proposed. According to Hauptfleisch, pseudotumors appearing with metal-metal pairings can be categorized into three types [34]:

- Type 1: cystic, wall $\leq 3 \mathrm{~mm}$, usually minor symptoms

- Type 2: cystic, wall > $3 \mathrm{~mm}$

- Type 3: primarily solid with the highest revision rate It is the consistency of the pseudotumor rather than its size alone that determine the signs and symptoms [34].

\section{Luxations}

Recurring luxation of hip TEP ( $\bullet$ Fig. $\mathbf{1 2 k}, \mathrm{I}$ ) is a complication that has decreased in incidence since the introduction of prosthesis planning involving restoration of the patientspecific anatomy. It can be caused by incorrect cup position (excessively high inclination angle, incorrect version (with correspondingly different frequency depending on surgical access pathway used)) as well as incorrect shaft position (excessive/insufficient antetorsion) [29]. Besides eliminating the cause, recurring luxation following hip total endoprosthesis can be treated by lengthening the prosthesis neck to create more tension on the soft tissues or using an inlay with a cambered cup rim [33]. Enlarging the diameter of the prosthetic head increases the degree of the freedom of motion of the prosthesis to the effect that the prosthesis 

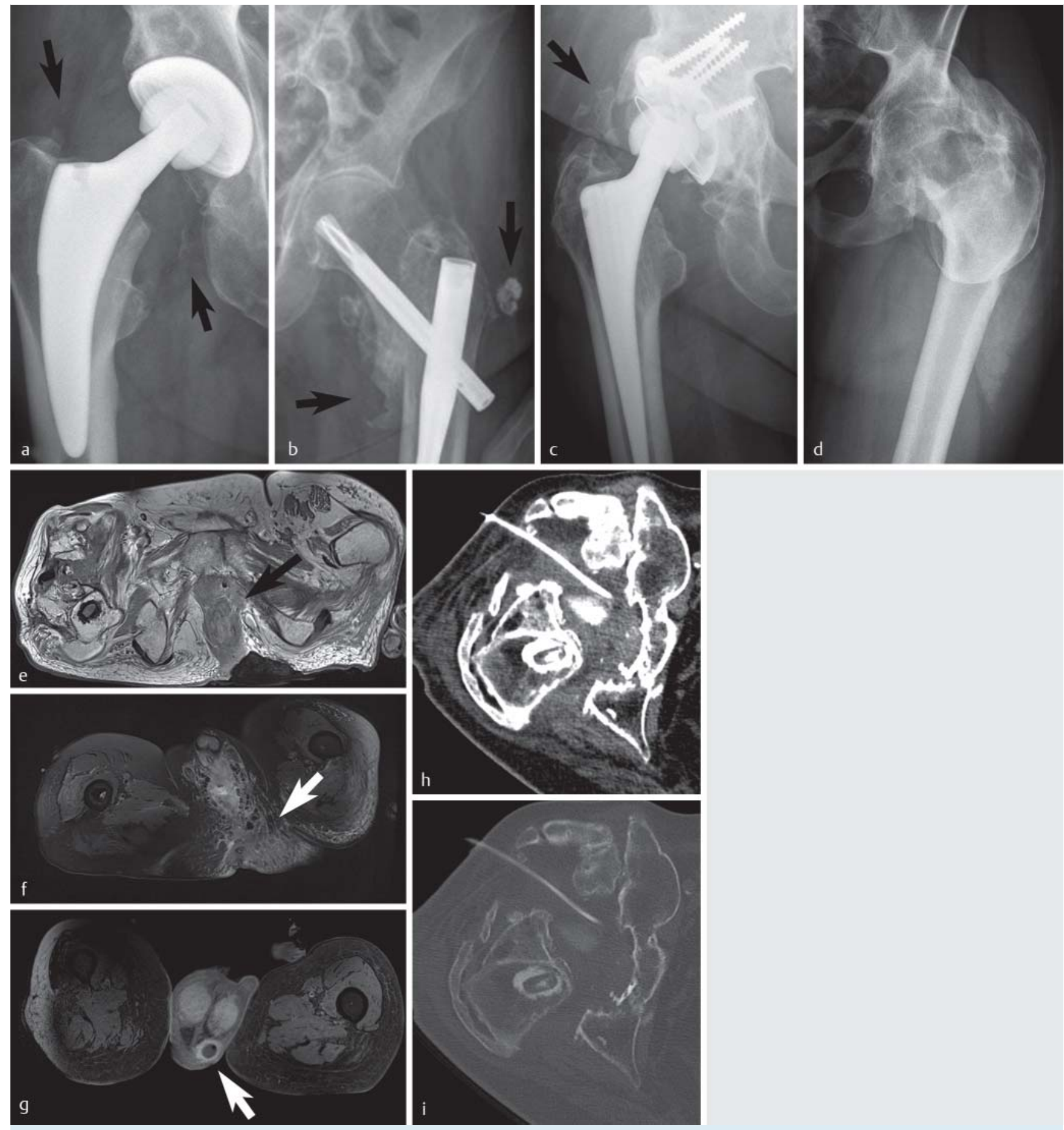

IV with ankylosis of the left hip joint d. 64-year-old, paraplegic male patient

Fig. 13 Illustration of various Brooker grades. Brooker grade I with small bone islets within the periarticular soft tissue a. Grade II with bone islets within the soft tissue and a bone spur arising from the femur and with more than $1 \mathrm{~cm}$ distance $\mathbf{b}$. Grade III with bone spurs emanating from femur and pelvis and with less than $1 \mathrm{~cm}$ between the opposing spurs $\mathrm{c}$. Brooker grade

neck does not strike against the rim of the cup (impingement) and thereby damage the cup or lever the prosthesis head out of the cup (luxation, subluxation) [29, 33]. The larger the prosthesis head, the lower the risk of luxation.

\section{Heterotopic ossifications (PAO)}

While the rate of incidence of heterotopic ossifications is very high especially over the long term at $40-50 \%$, it is with Girdlestone arthroplasty on the right side. MRI shows extensive decubitus with scrotal abscess expansion (e, T1-w, f, T2-w fs, g, contrast-enhanced $\mathrm{T} 1-\mathrm{w} \mathrm{fs}$, each in transverse orientation). Hip joint empyema of the right side was treated through CT-guided external drainage $\mathbf{h}, \mathbf{i}$. 
nounced PAOs following primary implantations have thus become less common. According to Brooker, heterotopic ossification in the hip is categorized into four grades ( $\bullet$ Fig. 13) [44]:

- Grade I - bone islets in soft tissue

- Grade II - osteophytes emanating from the femur or pelvis

- Grade III - same as grade II but with bone spurs being spaced less than $1 \mathrm{~cm}$ apart

- Grade IV - complete boney ankyloses.

\section{Metal artefacts in cross-sectional imaging}

In MRI, signal dropouts and noise at the phase interfaces of primarily magnetic materials (e.g. metal) are referred to as susceptibility artefacts. While in principle these can appear with any pulse sequence, spin echo sequences are less sensitive in this area owing to their pulse characteristics. In routine diagnostic testing, artifacts can be reduced through the following measures:

- Using spin echo and turbo spin echo sequences instead of gradient echo (GRE) sequences as well as using STIR sequences instead of frequency-selective techniques for fat suppression

- Interchanging phase and frequency direction

- Imaging with higher sensitivity bandwidth, if applicable orienting the longitudinal axis of the metal implant along the primary direction of the magnetic field

- Using modern artifact-reducing sequences, e.g. SEMAC (slice-encoding for metal artifact correction) or MARVRIC (multiple-acquisition variable-resonance imaging combination; GE Healthcare, Milwaukee, WI) [40, 42].

CT involves primarily hardening artifacts along metallic non-radiopaque materials. For iterative reconstruction there are already commercially available algorithms for these cases (e.g. O-MAR, Metal Artifact Reduction for Orthopedic Implants) [45]. With this technique, the first, uncorrected image is reconstructed and regularized, before then being compared with the gathered raw data. As long as these fail to match, other reconstructions are generated, thus constituting what is referred to as filtered back-projection. Image quality increases with the number of reconstructions. However, the disadvantage of this technique is the increased radiation dose required. Even CT scanning itself can considerably reduce artifacts through dual-energy techniques, allowing the same bodily region to be examined at two different energy levels [40].

\section{Summary and core statements on imaging after hip en- doprosthesis}

Problems occurring over the course of time with hip endoprostheses must be addressed through imaging as described below. Projection radiography examinations constitute the basic diagnostic means for evaluating implant position and detecting periprosthetic fractures, any loosening or soft tissue ossification. Computed tomography is the primary diagnostic method to enlist when radiographs yield unclear findings and periprosthetic fractures are suspected [29]. Modern prostheses such as those made of titanium alloys generate few artifacts. Sonography is suited for detecting or excluding joint effusion or postoperative seroma, while allowing direct fluid puncture and draining [25, 29]. MRI is indicated for suspected infection or tumor recur- rence in the case of tumor prostheses and pseudotumors. It is important that many clinical questions can be answered through thorough evaluation of conventional radiographs over the course of time. In this process it is also imperative that the entire prosthesis is imaged completely.

\section{References}

1 Funk JF, Lebek S. Epiphyseolysis capitis femoris. Neue Aspekte in Diagnostik und Therapie. Orthopäde 2014; 43: 742 - 749

2 Graf R. Radiologisch-orthopädische Anforderungsprofile bei der kindlichen Hüftdysplasie, der Koxitis und der Epiphyseolysis capitis femoris. Radiologe 2002; 42: 467-473

3 Breusch S, Mau H, Sabo D et al. Untere Extremität. In: Breusch S, Clarius M, Mau H. et al. (eds). Klinikleitfaden Orthopädie Unfallchirurgie. 7. Aufl. München: Elsevier; 2013: 429-562

4 Kramer J, Hofmann S, Scheurecker A et al. Morbus Perthes. Radiologe 2002; 42: 432-439

5 von Stillfried E, Weber MA. Aseptische Osteonekrosen bei Kindern und Jugendlichen. Orthopäde 2014; 43: 750 - 757

6 Multerer C, Döderlein L. Angeborene Hüftdysplasie und -luxation. Bewährte und neue Verfahren in Diagnostik und Therapie. Orthopäde 2014; 43: 733-741

7 Gould SW, Grissom LE, Niedzielski A et al. Protocol for MRI of the hips after spica cast placement. J Pediatr Orthop 2012; 32: 504-509

8 Steppacher SD, Tannast M, Siebenrock KA. Labrumläsionen des Hüftgelenks. Orthopädie und Unfallchirurgie up2date 2008; 3: 215-232

9 Kramer J, Scheurecker G, Scheurecker A et al. Hüftkopfnekrose. Radiologe 2009; 49: 410-418

10 Mattes T, Fraitzl C, Ostertag 0 et al. Differentialdiagnosen der aseptischen Hüftkopfnekrose. Artikulärer Leistenschmerz des Erwachsenen. Orthopäde 2007; 36: 414-422

11 Carty FL, Cashman JP, Parvizi J et al. Imaging of the postoperative hip. Semin Musculoskelet Radiol 2011; 15: 357-371

12 Fairbank AC, Bhatia D, Jinnah RH et al. Long-term results of core decompression for ischaemic necrosis of the femoral head. J Bone Joint Surg Br 1995; 77: 42 - 49

13 Karantanas AH. Acute bone marrow edema of the hip: role of MR imaging. Eur Radiol 2007; 17: 2225 - 2236

14 Vande Berg BC, Lecouvet FE, Koutaissoff S et al. Bone marrow edema of the femoral head and transient osteoporosis of the hip. Eur J Radiol 2008; 67: $68-77$

15 Mamisch TC, Werlen S, Zilkens $C$ et al. Radiologische Diagnose des femoroacetabularen Impingements. Radiologe 2009; 49: 425-433

16 Reid GD, Reid CG, Widmer $N$ et al. Femoroacetabular impingement syndrome: an underrecognized cause of hip pain and premature osteoarthritis? J Rheumatol 2010; 37: 1395-1404

17 Räuchle M, Cemerka M, Eibenberger B et al. Arthrose - update 2012. Radiologe 2012; 52: 149-155

18 Czerny C, Oschatz E, Neuhold A et al. MR Arthrograpie des Hüftgelenkes. Radiologe 2002; 42: 451-456

19 Petersilge CA. MR arthrography for evaluation of the acetabular labrum. Skeletal Radiol 2001; 30: 423-430

20 Llopis E, Fernandez E, Cerezal L. MR and CT arthrography of the hip. Semin Musculoskelet Radiol 2012; 16: 42 - 56

21 Nötzli HP, Wyss TF, Stoecklin CH et al. The contour of the femoral headneck junction as a predictor for the risk of anterior impingement. J Bone Joint Surg Br 2002; 84: 556-560

22 Ganz R, Gill TJ, Gautier E et al. Surgical dislocation of the adult hip a technique with full access to the femoral head and acetabulum without the risk of avascular necrosis. J Bone Joint Surg Br 2001; 83: $1119-1124$

23 Byrd JW. Hüftarthroskopie. Portaltechnik und arthroskopische Anatomie. Orthopäde 2006; 35: $41-53$

24 Botser IB, Smith TW Jr, Nasser $R$ et al. Open surgical dislocation versus arthroscopy for femoroacetabular impingement: a comparison of clinical outcomes. Arthroscopy 2011; 27: 270-278

25 Barron D. CT and MRI of hip arthroplasty. Clin Radiol 2007; 62: 1172 1173

26 Pfeil J, Höhle P, Rehbein P. Bilateraler endoprothetischer Ersatz am Hüft- oder Kniegelenk. Dtsch Arztebl Int 2011; 108: 463 - 468

27 Richter-Kuhlmann EA. Hochschulmedizin: Balanceakt Indikationsstellung. Dtsch Arztebl 2013; 110: B - 749 
28 Mayerhoefer ME, Fruhwald-Pallamar J, Czerny C. Bildgebung bei Hüftgelenkendoprothesen. Radiologe 2009; 49: 419-424

29 Miller TT. Imaging of hip arthroplasty. Eur J Radiol 2012; 81: $3802-$ 3812

30 Hailer NP, Garellick G, Kärrholm J. Uncemented and cemented primary total hip arthroplasty in the Swedish Hip Arthroplasty Register. Acta Orthop 2010; 81: 34-41

31 Azegami S, Gurusamy KS, Parker MJ. Cemented versus uncemented hemiarthroplasty for hip fractures: a systematic review of randomised controlled trials. Hip Int 2011; 21: 509-517

32 Abdulkarim A, Ellanti P, Motterlini N et al. Cemented versus uncemented fixation in total hip replacement: a systematic review and meta-analysis of randomized controlled trials. Orthop Rev (Pavia) 2013; 5: e8. DOI: $10.4081 /$ or.2013.e8

33 Duparc J. (ed). Chirurgische Techniken in Orthopädie und Traumatologie. München: Urban \& Fischer Verlag; 2005

34 Hauptfleisch J, Pandit H, Grammatopoulos G et al. A MRI classification of periprosthetic soft tissue masses (pseudotumours) associated with metal-on-metal resurfacing hip arthroplasty. Skeletal Radiol 2012; 41: $149-155$

35 Parsons TM, Satchithananda K, Berber $R$ et al. Magnetresonanztomographische Untersuchungen bei Problemen mit Metall-auf-Metall-Implantaten. Orthopäde 2013; 42: 629-636

36 Cahir JG, Toms AP, Marshall TJ et al. CT and MRI of hip arthroplasty. Clin Radiol 2007; 62: 1163 -1171
37 Jesse MK, Petersen B, Strickland C et al. Normal anatomy and imaging of the hip: emphasis on impingement assessment. Semin Musculoskelet Radiol 2013; 17: 229-247

38 Tschauner C, Fock CM, Hofmann S et al. Rotationsfehler des Hüftgelenks. Radiologe 2002; 42: 457-466

39 Pradhan R. Planar anteversion of the acetabular cup as determined from plain anteroposterior radiographs. J Bone Joint Surg Br 1999; 81: $431-435$

40 Agten CA, Sutter R, Pfirrmann CWA. CT und MRT der Hüftprothese. Radiologe 2014; 54: 715-726

41 Hendrich C, Sauer $U$, Albrecht T et al. Subsidence of titanium straight stems in combination with highly viscous bone cement. Int Orthop 2005; 29: $96-100$

42 Hayter CL, Koff MF, Potter HG. Magnetic resonance imaging of the postoperative hip. J Magn Reson Imaging 2012; 35: 1013-1025

43 Ostlere $S$. How to image metal-on-metal prostheses and their complications. Am J Roentgenol 2011; 197: 558-567

44 Brooker AF, Bowerman JW, Robinson RA et al. Ectopic ossification following total hip replacement. Incidence and a method of classification. J Bone Joint Surg Am 1973; 55: 1629-1632

45 Kidoh M, Nakaura T, Nakamura S et al. Reduction of dental metallic artefacts in CT: value of a newly developed algorithm for metal artefact reduction (O-MAR). Clin Radiol 2014; 69: e11 -e16 